\title{
THE SHIMURA CURVE OF DISCRIMINANT 15 AND TOPOLOGICAL AUTOMORPHIC FORMS
}

\author{
TYLER LAWSON \\ Department of Mathematics, University of Minnesota, 206 Church Street SE, Minneapolis, \\ MN 55414, USA; \\ email: tlawson@math.umn.edu
}

Received 17 January 2013; accepted 17 January 2015

\begin{abstract}
We find defining equations for the Shimura curve of discriminant 15 over $\mathbb{Z}[1 / 15]$. We then determine the graded ring of automorphic forms over the 2-adic integers, as well as the higher cohomology. We apply this to calculate the homotopy groups of a spectrum of 'topological automorphic forms' associated to this curve, as well as one associated to a quotient by an AtkinLehner involution.
\end{abstract}

2010 Mathematics Subject Classification: 55P42 (primary); 11F23, 11G18, 14G35, 55P43 (secondary)

\section{Introduction}

A generalized cohomology theory $E$ associates to each space $X$ a sequence of abelian groups $E^{n}(X)$, often equipped with extra structure such as a graded multiplication. These are required to satisfy the Eilenberg-Steenrod axioms, and this alone implies that there is a natural Atiyah-Hirzebruch spectral sequence

$$
H^{s}\left(X ; E^{t}(*)\right) \Rightarrow E^{s+t}(X)
$$

with reasonable convergence properties when $X$ has the homotopy type of a CWcomplex.

In a coarse sense, this tells us that $E^{*}(X)$ combines the cohomological data of $X$ and the $E$-cohomology of a point in some way. However, the spectral sequence is

(C) The Author 2015. This is an Open Access article, distributed under the terms of the Creative Commons Attribution licence (http://creativecommons.org/licenses/by/3.0/), which permits unrestricted re-use, distribution, and reproduction in any medium, provided the original work is properly cited. 
the first step in an iterative calculation, and much deeper information is required to complete it: in particular, we need to find differentials and solve hidden extensions in this spectral sequence. In a local sense these depend on $X$ : if $X$ is a CWcomplex, this information is determined by $X$ and the attaching maps for the cells of $X$. In a global sense these depend on $E$ : this information is determined by an intricate web of connective tissue between the groups $E^{t}(*)$, expressible in terms of cohomology operations, secondary operations, and so on.

It has been an active research topic to determine all the data necessary to take a graded abelian group, or a graded ring, and lift it to a generalized cohomology theory. Quillen showed that for many multiplicative cohomology theories there is a theory of Chern classes, and the formula for the first Chern class of a tensor product of line bundles gives $E^{*}$ a natural formal group law [Qui69]. Landweber showed that the converse holds in many circumstances: given a graded-commutative ring with an appropriate type of graded formal group law, one can realize it by a generalized cohomology theory [Lan76].

More recently, Lurie announced a very strongly functorial result of this converse type, generalizing work of Hopkins, Miller, and others. It requires more input: instead of just a formal group law, Lurie's result requires an extension of it to a $p$-divisible group satisfying a version of the Serre-Tate property [Goe09]. This has been exploited to construct generalized cohomology theories attached to certain moduli of abelian varieties, under the general header of topological automorphic forms [BL10]. Lurie's theorem takes a scheme (or stack) with such a $p$-divisible group and equips its étale site with a sheaf of $E_{\infty}$ ring spectra (the homotopy-theoretic analog of a sheaf of commutative differential graded algebras).

This gives us an abundance of new objects in homotopy theory, with each described by purely algebro-geometric data. To bring ourselves back down to earth, we must understand the consequences of what we have done. The data itself determines many cohomology theories $E$ and their coefficient rings; about the connective tissue it gives not much direct information.

Shimura curves have provided an interesting test case. These parameterize twodimensional abelian varieties with an action of a quaternion algebra, and they share many formal similarities with the moduli of elliptic curves. They have been harder to cohomologically analyze than the moduli of elliptic curves, but in many cases their images in homotopy theory have provided similar answers. In [HL10], the resulting cohomology theories were analyzed for the Shimura curves of discriminants 6,10 , and 14 . The most mysterious aspect appeared with the curve of discriminant 10 . This curve has a very different geometry than the moduli of elliptic curves, but after 3-adic completion the Shimura curve and the modular curve have associated cohomology theories that behave in an identical fashion so far as investigations have revealed. 
The program of this paper is to study the Shimura curve of discriminant 15 and its relation to homotopy theory. The prime 2 does not divide 15 , and as a result this is the first Shimura curve where 2-primary information in stable homotopy theory can be extracted. The path we will follow is similar to that in [HL10]: we describe a moduli object, determine its cohomology, and find the coefficient ring of the resulting cohomology theory by a spectral sequence calculation.

This results in new questions. The final calculations in homotopy theory are very similar to the calculations of Mahowald and Rezk for topological modular forms with level $\Gamma_{0}(3)$ structure [MR09] - so similar that Figures 12.1 and 12.3 could easily be used as references for the calculations that appeared in their paper. At the prime 2, the coefficient ring for the Shimura curve breaks up as isomorphic to a direct sum of two pieces: a coefficient ring for topological modular forms with level $\Gamma_{0}(3)$ structure, and a certain module over it. Moreover, the Shimura curve has an action of $\mathbb{Z} / 2$, and the quotient corresponds to a cohomology theory with a new coefficient ring, now an extension of the coefficient ring for topological modular forms with level $\Gamma_{0}(3)$ structure. This, again, occurs despite the lack of obvious geometric connection between these moduli. It is not clear if something fundamental is guiding these connections, or if these are merely coincidences in low degrees.

We will now give a detailed outline, beginning with a more exact description of the objects under consideration.

There is a four-dimensional division algebra $D$ over $\mathbb{Q}$, generated by elements $x$ and $y$ satisfying

$$
x^{2}=-3, \quad y^{2}=5, \quad x y=-y x .
$$

This is uniquely characterized by the requirement that $D \otimes \mathbb{Q}_{p} \cong M_{2}\left(\mathbb{Q}_{p}\right)$ precisely for primes $p \neq 3,5$ (in other words, it has discriminant 15). In particular, there is an isomorphism

$$
\tau: D \otimes \mathbb{R} \cong M_{2}(\mathbb{R}) .
$$

On $D$ there is a reduced norm $N(a+b x+c y+d x y)=a^{2}+3 b^{2}-5 c^{2}-15 d^{2}$ which is multiplicative, and under $\tau$ it corresponds to the determinant.

Within $D$ there is also a subring

$$
\Lambda \cong \mathbb{Z}\langle\omega, y\rangle /\left(\omega^{2}+\omega+1, y \omega=\omega^{2} y\right),
$$

generated by $y$ and $\omega=(-1+x) / 2$. The ring $\Lambda$ is maximal among finitely generated submodules closed under the multiplication (a maximal order), and any other such subring is conjugate to $\Lambda$ [Eic38]. We obtain an embedding of the norm-1 subgroup:

$$
\tau: \Lambda^{N=1} \rightarrow S L_{2}(\mathbb{R})
$$


This gives the action of the norm- 1 elements of $\Lambda$ on the complex upper halfplane $\mathcal{H}$. The quotient orbifold $\mathcal{H} / \Lambda^{N=1}=\mathcal{X}_{\mathbb{C}}^{D}$ is called the complex Shimura curve of discriminant 15 , and it is a parameterizing object for two-dimensional complex abelian varieties $A$ equipped with an action of $\Lambda$ (sometimes called fake elliptic curves). The orbifold structure reflects the fact that such objects $A$ often possess automorphisms.

The object $\mathcal{X}_{\mathbb{C}}^{D}$, through this interpretation as a parameterizing object, has an algebraic lift. Over $\mathbb{Z}[1 / 15]$, there is a stack $\mathcal{X}^{D}$ such that maps $S \rightarrow \mathcal{X}^{D}$ parameterize two-dimensional abelian schemes $A / S$ with an action of $\Lambda$. There is an underlying coarse moduli scheme $X^{D}$ which is a smooth curve over $\mathbb{Z}[1 / 15]$ [Mor81]. The first goal of this paper is purely algebraic: it is to determine defining equations for $X^{D}$. As in [HL10], this builds on previous work of Kurihara [Kur79] and Elkies [Elk98].

The second goal concerns automorphic forms, and it requires us to extend from $\mathbb{Z}[1 / 15]$ to the ring $\mathbb{Z}_{2}$ (though statements could be made over general rings $R$ such that $\Lambda \otimes R \cong M_{2}(R)$ ). Letting $\mathcal{A} / \mathcal{X}^{D}$ be the universal abelian scheme, the action of $\Lambda \otimes \mathbb{Z}_{2} \cong M_{2}\left(\mathbb{Z}_{2}\right)$ on the two-dimensional relative cotangent space of $\mathcal{A}_{\mathbb{Z}_{2}}$ at the identity splits it into two isomorphic one-dimensional summands. This summand is a line bundle $\omega$ on $\mathcal{X}_{\mathbb{Z}_{2}}^{D}$, and the sections of $\omega^{\otimes t}$ are automorphic forms of weight $t$ on $\mathcal{X}_{\mathbb{Z}_{2}}^{D}$. The second goal of this paper is also algebraic: it is to determine the cohomology groups $H^{s}\left(\mathcal{X}_{\mathbb{Z}_{2}}^{D} ; \omega^{\otimes t}\right)$. In particular, when $s=0$ this is a graded ring of automorphic forms over $\mathbb{Z}_{2}$.

We next 2-adically complete and study $\left(\mathcal{X}^{D}\right)_{2}^{\wedge}$, a formal parameter object living over $\mathbb{Z}_{2}$. In this case, the actions of $\Lambda$ on the two-dimensional formal group $\widehat{\mathcal{A}}$ and the two-dimensional 2-divisible group $\mathcal{A}\left[2^{\infty}\right]$ factor through $\Lambda \otimes \mathbb{Z}_{2}$. This splits the 2-divisible group into two isomorphic one-dimensional summands, and similarly for the formal group. The aforementioned theorem of Lurie then lifts this formal group data to a derived structure sheaf $\mathcal{O}^{d e r}$ of $E_{\infty}$ ring spectra (see [HL10, Section 2.6]), and the homotopy groups are determined by a cohomology spectral sequence

$$
H^{s}\left(U ; \omega^{\otimes t}\right) \rightarrow \pi_{2 t-s} \Gamma\left(U, \mathcal{O}^{d e r}\right) .
$$

In particular, we can define $\operatorname{TAF}^{D}=\Gamma\left(\left(\mathcal{X}^{D}\right)_{2}^{\wedge}, \mathcal{O}^{\text {der }}\right)$ as a global section object. The third goal of this paper is to determine the homotopy groups of this spectrum. As stated, the calculations are similar to those of Mahowald and Rezk. The main difference is that an extra summand occurs in the computation (Figures 12.2 and 12.4), which carries out a twist of the Mahowald-Rezk calculation.

Finally, there is an Atkin-Lehner involution $w_{15}$ on $\mathcal{X}^{D}$, whose effect is to take an abelian variety with $\Lambda$-action and 'twist' the action by conjugating with $x y$. Since -15 has a 2 -adic square root, the action of $w_{15}$ lifts to the 2-divisible group (see 10.1), and so we get an action of $\mathbb{Z} / 2$ on $\mathrm{TAF}^{D}$. The final goal of this 
paper is to determine the homotopy groups of this homotopy fixed-point spectrum, which could be viewed as the global section object of an extension of $\mathcal{O}^{\text {der }}$ to the quotient stack $\mathcal{X}^{D} / w_{15}$. This calculation is, again, similar to the Mahowald-Rezk calculation, but this time it adds an ideal carrying out two copies of the homotopy fixed-point spectral sequence for $K O$.

The exact connection of $\mathrm{TMF}_{0}(3)$ with $\mathrm{TAF}^{D}$ on the spectrum level remains unclear. Both map to a homotopy fixed-point spectrum $E O(G)$ associated to a finite subgroup $G$ of the extended Morava stabilizer group, but this alone does not support the degree of connection that is visible in homotopy theory.

\section{Complex uniformization}

The Shimura curve $X^{D}$ of discriminant 15 is of genus 1 , and it has two elliptic points of order 3 .

In order to determine a complex uniformization of the curve, we first fix an embedding $\tau$ of the division algebra $D$ into $M_{2}(\mathbb{R})$. This differs from that in $[\mathrm{AB04}, 5.5]$, and the resulting fundamental domain differs from that in [AB04, Figure 5.3], which was determined by Michon [Mic81] and pictured in [Vig80, IV. Section 3.C].

Let $\tau: D \rightarrow M_{2}(\mathbb{R})$ be defined on the generators of Equation (1.1) by

$$
x \mapsto\left[\begin{array}{cc}
0 & \sqrt{3} \\
-\sqrt{3} & 0
\end{array}\right], \quad y \mapsto\left[\begin{array}{cc}
0 & \sqrt{5} \\
\sqrt{5} & 0
\end{array}\right], \quad x y \mapsto\left[\begin{array}{cc}
\sqrt{15} & 0 \\
0 & -\sqrt{15}
\end{array}\right] .
$$

This determines the action of $\Lambda^{\times}$on $\mathbb{C} \backslash \mathbb{R}$, with quotient the complex Shimura curve $X_{\mathbb{C}}^{D}$. Write $\mathcal{H}$ for the upper half-plane.

We have norm- 1 elements in $\Lambda$ :

$$
\omega=\frac{-1+x}{2}, \quad h=4+x y, \quad \gamma=4+5 \omega^{2}-2 y .
$$

The first rotates by $2 \pi / 3$ around an elliptic point $i \in \mathcal{H}$, while the element $4+x y$ is a 'principal homothety' $h: z \mapsto(4+\sqrt{15})^{2} z$.

We also define elements

$$
\tilde{w}_{3}=x, \quad \tilde{w}_{5}=5+2 y, \quad \tilde{w}_{15}=5 x+2 x y .
$$

These elements have norms 3,5, and 15, respectively. They thus act on the upper half-plane quotient as lifts of the Atkin-Lehner involutions $w_{3}, w_{5}$, and $w_{15}$, respectively. The element $\tilde{w}_{5}$ is a hyperbolic translation stabilizing the circle of radius 1 , while $\tilde{w}_{3}$ is an involution about this circle that fixes $i$. 


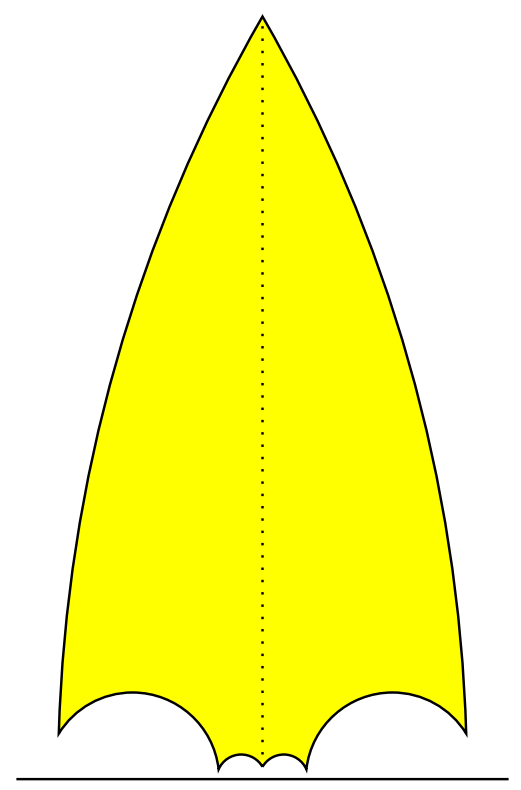

Figure 2.1. Fundamental domain for $\mathcal{X}_{\mathbb{C}}^{D}$.

We note that, in $\Lambda$, there is an identity

$$
5 h=\left(\omega^{2} \tilde{w}_{5} \omega^{2}+5\right) y .
$$

Together with the fact that $y$ commutes with $\tilde{w}_{5}$ and conjugate-commutes with $\omega$, this shows that

$$
\left(\omega^{2} \tilde{w}_{5} \omega^{2}\right) h=h\left(\omega \tilde{w}_{5} \omega\right)
$$

PROPOSITION 2.1. There exists a hyperbolic hexagon (Figure 2.1) which is a fundamental domain for the action of $\Lambda^{N=1} /\{ \pm 1\}$ on $\mathcal{H}$. It has the following six elliptic points as vertexes:

$$
\begin{array}{lll}
v_{1}=h \omega \tilde{w}_{5} i & v_{2}=h i & v_{3}=h \omega^{2} \tilde{w}_{5}^{-1} i \\
v_{6}=\omega^{2} \tilde{w}_{5} i & v_{5}=i & v_{4}=\omega \tilde{w}_{5}^{-1} i
\end{array}
$$

This domain is symmetric about the imaginary axis, and the edges are identified via

$$
\begin{gathered}
h \omega^{2}\left(\overrightarrow{v_{5} v_{6}}\right)=\overrightarrow{v_{2} v_{1}}, \\
h \omega\left(\overrightarrow{v_{5} v_{4}}\right)=\overrightarrow{v_{2} v_{3}}, \\
\gamma\left(\overrightarrow{v_{4} v_{3}}\right)=\overrightarrow{v_{6} v_{1}} .
\end{gathered}
$$


Proof. As elliptic points are preserved by the action of $\Lambda$, the given vertexes are all elliptic points in $\mathcal{H}$. We note that the operator $\omega^{2} \tilde{w}_{5} \omega^{2}$ takes $v_{4}$ to $v_{5}$ and $v_{5}$ to $v_{6}$; Equation (2.4) implies that it also takes $v_{3}$ to $v_{2}$ and $v_{2}$ to $v_{1}$.

We will now show that $v_{1}, \ldots, v_{6}$ are the vertexes, indexed in clockwise order, of a fundamental domain for the action of $\Lambda^{N=1}$. The resulting curve is of genus 1 .

The geodesics $\omega\left(\vec{v}_{5} \vec{v}_{6}\right)$ and $\omega^{2}\left(\vec{v}_{5} v_{4}\right)$ are along the unit circle. Therefore, the original geodesics $\overrightarrow{v_{5} v_{6}}$ and $\vec{v}_{5} v_{4}$ make angles of $\pi / 6$ with the imaginary axis, represented by $\overrightarrow{v_{5} v_{2}}$. Similarly, $\overrightarrow{v_{2} v_{1}}$ and $\overrightarrow{v_{2} v_{3}}$ also make angles of $\pi / 6$ with $\overrightarrow{v_{5} v_{2}}$. The hyperbolic volume is therefore $8 \pi / 3$, which is the volume of $X^{D}$. Therefore, once we have shown that the geodesic edges are identified, this must be a fundamental domain.

The following identifications of geodesics are verified by checking that they have the correct effect on endpoints.

- The transformation $a=h \omega^{2}$ takes $\overrightarrow{v_{5} v_{6}}$ to $\overrightarrow{v_{2} v_{1}}$.

- The transformation $b=h \omega$ takes $\overrightarrow{v_{5} v_{4}}$ to $\overrightarrow{v_{2} v_{3}}$.

- The transformation $\gamma=4+5 \omega^{2}-2 y$, which is also realized by the element

$$
\left(\omega^{2} \tilde{w}_{5} \omega^{2}\right)^{2}=5\left(4+5 \omega^{2}-2 y\right)
$$

takes $\overrightarrow{v_{4} v_{3}}$ to $\overrightarrow{v_{6} v_{1}}$.

This fundamental domain lets us produce a presentation of the group $\Lambda^{N=1} /\{ \pm 1\}$, which is the fundamental group of the associated orbifold.

COROLlaRY 2.2. There is a presentation

$$
\Lambda^{N=1} /\{ \pm 1\}=\left\langle h, \gamma, \omega \mid \omega^{3}=\left(\omega^{2} h^{-1} \gamma h \omega^{2} \gamma^{-1}\right)^{3}=1\right\rangle .
$$

Proof. The fundamental domain gives us the generators $a=h \omega^{2}, b=h \omega$, and $\gamma$, and relations

$$
\left(b^{-1} a\right)^{3}=\left(b^{-1} \gamma a \gamma^{-1}\right)^{3}=1 .
$$

By switching generators to $h, \omega$, and $\gamma$, we obtain the desired presentation.

\section{Complex multiplication}

In this section we review results of Elkies on the structure of the Shimura curve $X_{\mathbb{Q}}^{D}$ and its quotients. All of the results in this section, and in particular the complex multiplication (CM) points, are taken directly from [Elk98, Section 5.2]. 
The quotient $\left(X_{\mathbb{Q}}^{D}\right)^{*}$ by the full group of Atkin-Lehner involutions is of genus zero, with one elliptic point $P_{6}$ of order $6(\mathrm{CM}$ discriminant -3$)$ and three elliptic points $P_{2}, P_{2}^{\prime}$, and $P_{2}^{\prime \prime}$ of order 2 (CM discriminants $-12,-15$, and -60 , respectively).

There is thus a unique isomorphism $t:\left(X^{D}\right)^{*} \rightarrow \mathbb{P}^{1}$ so that $t\left(P_{6}\right)=\infty$, $t\left(P_{2}\right)=0$, and $t\left(P_{2}^{\prime \prime}\right)=1$. Elkies showed that this coordinate satisfies $t\left(P_{2}^{\prime}\right)=81$, and that (among others) there is a further rational point with $t$-coordinate -27 and CM discriminant -7 [Elk98, Table 6].

We now examine the Atkin-Lehner involutions $w_{3}, w_{5}$, and $w_{15}$. We recall that a point of $X^{D}$ is fixed by $w_{d}$ if and only if it parameterizes a surface with a $\Lambda$ linear endomorphism $t$ satisfying $t^{2}+m t+d=0$; this means that it has complex multiplication by the splitting field of this polynomial, and that this splitting field also splits $D$.

The involution $w_{5}$ fixes only those points which correspond to points with an action by $\mathbb{Z}[\sqrt{-5}]$. However, since 3 splits in $\mathbb{Q}(\sqrt{-5})$, this field does not split $D$, and so the involution $w_{5}$ has no fixed points.

Similarly, the involution $w_{3}$ on $X^{D}$ fixes only those points parameterizing objects with an action of $\mathbb{Z}[\sqrt{-3}]$. These are precisely the $\mathrm{CM}$ points with discriminants -3 and -12 , and hence the preimages of $P_{6}$ and $P_{2}$.

Finally, the involution $w_{15}$ fixes two points with an action of $\mathbb{Z}[\sqrt{-15}]$. These must have CM discriminant -15 or -60 , and so are the preimages of $P_{2}^{\prime}$ and $P_{2}^{\prime \prime}$.

The curve $\left(X_{\mathbb{Q}}^{D}\right) / w_{3}$ has exactly one preimage each of $P_{2}$ and $P_{6}$. The two preimages of $P_{2}^{\prime}$ are complex conjugate and their coordinates must generate the Hilbert class field of $\mathbb{Q}(\sqrt{-15})$ by work of Shimura; this class field is $\mathbb{Q}(\sqrt{-15}$, $\sqrt{-3})$. The preimages are thus defined over $\mathbb{Q}(\sqrt{-3})$, and we can define a unique Galois-invariant coordinate $s$ such that $s\left(P_{6}\right)=\infty, s\left(P_{2}\right)=0$, and the preimages of $P_{2}^{\prime}$ are taken to $\pm \sqrt{-3}$. This coordinate satisfies $t=-3 s^{2}$.

Then [Elk98, (76)] shows that there is a coordinate $y$ on $X^{D} / w_{15}$ so that

$$
y^{2}=-\left(3 s^{2}+1\right)\left(s^{2}+27\right)=-(1-t)(27-t / 3) .
$$

Together $s$ and $y$ generate the function field of the curve. By construction, $y$ is fixed by $w_{15}$ and negated by $w_{3}$ and $w_{5}$, while $s$ is fixed by $w_{3}$ and negated by $w_{5}$ and $w_{15}$.

\section{Rational uniformization}

In this section we review the structure of the rational Shimura curve $X_{\mathbb{Q}}^{D}$ and its quotients, continuing with the notation from the previous section. 
Proposition 4.1. The curves $\left(X_{\mathbb{Q}}^{D}\right) / w_{3}$ and $\left(X_{\mathbb{Q}}^{D}\right) / w_{15}$ are both isomorphic to $\mathbb{P}^{1}$, and the natural map from $X^{D}$ into their product is an embedding.

Proof. The map $X_{\mathbb{Q}}^{D} \rightarrow\left(X_{\mathbb{Q}}^{D}\right) / w_{3} \times\left(X_{\mathbb{Q}}^{D}\right) / w_{15}$ only fails to be at an embedding at places fixed by both $w_{3}$ and $w_{15}$, hence $w_{5}$.

The involution $w_{5}$, with no fixed points, acts as an order- 2 translation on the genus-one curve. The quotient $X^{D} / w_{5}$ is of genus one, and under the projection $X^{D} / w_{5} \rightarrow\left(X^{D}\right)^{*}$ there is a single preimage of $P_{6}$ which is an elliptic point of order 3. (The subspace of Figure 2.1 with positive real part is a fundamental domain for $X_{\mathbb{C}}^{D} / w_{5}$.)

The quotient curve $X^{D} / w_{3}$ is of genus zero with two elliptic points of order 6 (the preimages of $P_{6}$ ) and two of order 2 (the preimages of $P_{2}$ ). As $X^{D} / w_{3}$ is smooth of genus zero and has points defined over $\mathbb{Q}$, it is isomorphic to $\mathbb{P}^{1}$ over $\mathbb{Z}[1 / 15]$.

The quotient curve $X^{D} / w_{15}$ is also of genus zero, with one elliptic point of order 3 (the preimage of $P_{6}$ ) and four of order 2 (the preimages of $P_{2}^{\prime}$ and $P_{2}^{\prime \prime}$ ). Similarly, it is isomorphic to $\mathbb{P}^{1}$ over $\mathbb{Z}[1 / 15]$.

\section{PROPOSITION 4.2. There are meromorphic functions}

$$
u:\left(X_{\mathbb{Q}}^{D}\right) / w_{3} \stackrel{\sim}{\rightarrow} \mathbb{P}_{\mathbb{Q}}^{1} \quad \text { and } \quad v:\left(X_{\mathbb{Q}}^{D}\right) / w_{15} \stackrel{\sim}{\rightarrow} \mathbb{P}_{\mathbb{Q}}^{1}
$$

so that the resulting embedding $X_{\mathbb{Q}}^{D} \rightarrow \mathbb{P}_{\mathbb{Q}}^{1} \times \mathbb{P}_{\mathbb{Q}}^{1}$ has, as image, the (closure of the) set of solutions of

$$
\left(u^{2}+u+1\right)\left(v^{2}+v+1\right)=\frac{5}{9} .
$$

The Atkin-Lehner operator $w_{3}$ fixes $u$ and sends $v$ to $-1-v$, while $w_{15}$ fixes $v$ and sends $u$ to $-1-u$.

Proof. We will first change the coordinates $t, s$, and $y$, from the previous section, into new ones that will ultimately prove better behaved integrally.

Let $w=(t-1) / 4$ denote the 'equivalent, 2-adically good' coordinate Elkies describes on $\left(X_{\mathbb{Q}}^{D}\right)^{*}$. We also let $u=(s-3) / 6$ and $v=$ $(3 y-(t-81)) /(2(t-81))$ so that $t=4 w+1, s=6 u+3$, and $y=(4(2 v+1)(w-20)) / 3$. These still generate the function field.

The equation $t=-3 s^{2}$ is equivalent to $w=-27 u^{2}-27 u-7$.

The equation $y^{2}=-(1-t)(27-t / 3)$ becomes $v^{2}+v=(w-5) /(20-w)$, or $w=20-(15) /\left(v^{2}+v+1\right)$.

Putting these two equations for $w$ together, we find that $u$ and $v$ generate the function field of the curve $X_{\mathbb{Q}}^{D}$ and satisfy the formula of Equation (4.1). 
Together the coordinates $u$ and $v$ determine an embedding $X_{\mathbb{Q}}^{D} \rightarrow \mathbb{P}_{\mathbb{Q}}^{1} \times \mathbb{P}_{\mathbb{Q}}^{1}$ over $\mathbb{Q}$, representing the map $X_{\mathbb{Q}}^{D} \rightarrow X_{\mathbb{Q}}^{D} / w_{3} \times X_{\mathbb{Q}}^{D} / w_{15}$.

\section{CM points and integral uniformization}

We recall the intersection theory employed in [HL10], based on [KRY06]. Given an element $z \in \mathbb{C}$ generating a quadratic imaginary field, let $D_{z}$ be the divisor on $X_{\mathbb{Z}[1 / 15]}^{D}$ parameterizing points with complex multiplication by $z$. The following is derived by knowing that when two such divisors with distinct associated fields intersect, the resulting point has two distinct types of complex multiplication and thus must represent a supersingular point.

PROPOSITION 5.1. Let two orders be generated by elements $x$ and $y$ contained in nonisomorphic quadratic imaginary fields, with $d_{x}$ and $d_{y}$ their respective discriminants. If $D_{x} \cap D_{y}$ contains points in characteristic $p$, there must be an integer $m$ such that the quantity

$$
\Delta=(2 m+\operatorname{Tr}(x) \operatorname{Tr}(y))^{2}-\mathrm{N}(x) \mathrm{N}(y)
$$

produces Hilbert symbols

$$
\left(\Delta, d_{y}\right)_{q}=\left(\Delta, d_{x}\right)_{q}
$$

which are nontrivial precisely when $q \in\{p, \infty, 3,5\}$. (In particular, $\Delta$ must be negative.)

We now choose a point $Q$ on $X^{D} / w_{3}$ with $s$-coordinate 3 . This makes $t=-3 s^{2}$ equal -27 , which was already established to give it CM discriminant -7 .

Table 5.1 collects together the values of the coordinates $u$ and $v$ on the (preimages of) complex multiplication points discussed in the previous sections. Where not explicitly stated previously, these are explicitly derived from the equations relating these six coordinates. By convention, in the following table $\omega$ is the third root of unity $(-1+\sqrt{-3}) / 2$.

We thus consider the divisors on $X_{\mathbb{Z}[1 / 15]}$ with complex multiplication by $(-1+\sqrt{-3}) / 2$, by $(-1+\sqrt{-7}) / 2$, and by $(-1+\sqrt{-15}) / 2$. These have discriminants $-3,-7$, and -15 , respectively, and their divisors are associated to the preimages of the points $P_{6}, P_{2}^{\prime}$, and $Q$ in Table 5.1.

Proposition 5.2. The divisors associated to $P_{6}, P_{2}^{\prime}$, and $Q$ do not intersect on $X^{D}$. 
Table 5.1. Complex multiplication points.

\begin{tabular}{cccccccc}
\hline Point & CM disc & $t$ & $s$ & $y$ & $w$ & $u$ & $v$ \\
\hline$P_{6}$ & -3 & $\infty$ & $\infty$ & $\infty$ & $\infty$ & $\infty$ & $\omega$ \\
$P_{2}$ & -12 & 0 & 0 & $3 \sqrt{-3}$ & $\frac{-1}{4}$ & $\frac{-1}{2}$ & $\frac{-3+1 / \sqrt{-3}}{6}$ \\
$P_{2}^{\prime}$ & -15 & 81 & $3 \sqrt{-3}$ & 0 & 20 & $\omega$ & $\infty$ \\
$P_{2}^{\prime \prime}$ & -60 & 1 & $1 / \sqrt{-3}$ & 0 & 0 & $\frac{\omega-4}{9}$ & $\frac{-1}{2}$ \\
$Q$ & -7 & -27 & 3 & $12 \sqrt{-7}$ & -7 & 0 & $\frac{\sqrt{-7}-3}{6}$ \\
\hline
\end{tabular}

Table 5.2. Complex multiplication intersections.

\begin{tabular}{ccccc}
\hline Points & & $m$ & $\Delta$ & Nonvanishing Hilbert symbols \\
\hline$P_{6}$ & $Q$ & $0,-1$ & -20 & $\{5, \infty\}$ \\
& & $1,-2$ & -12 & $\{3, \infty\}$ \\
$P_{6}$ & $P_{2}^{\prime}$ & $0,-1$ & -44 & $\{11, \infty\}$ \\
& & $1,-2$ & -36 & $\{3, \infty\}$ \\
& & $2,-3$ & -20 & $\{5, \infty\}$ \\
$Q$ & $P_{2}^{\prime}$ & $0,-1$ & -104 & $\{13, \infty\}$ \\
& & $1,-2$ & -96 & $\{3, \infty\}$ \\
& & $2,-3$ & -80 & $\{5, \infty\}$ \\
& $3,-4$ & -56 & $\{7, \infty\}$ \\
& & $4,-5$ & -24 & $\{3, \infty\}$ \\
\hline
\end{tabular}

Proof. Table 5.2 summarizes the possible valid discriminants $\Delta$ from Equation (5.1) and the list of primes with nonvanishing Hilbert symbols from Equation (5.2) for all possible pairs.

As a result, none of these three divisors intersect at any prime in $\mathbb{Z}[1 / 15]$.

Proposition 5.3. The embedding $u \times v$ of Proposition 4.2 extends to an embedding $X^{D} \rightarrow \mathbb{P}^{1} \times \mathbb{P}^{1}$ over $\mathbb{Z}[1 / 15]$, giving $X^{D}$ the same defining equation integrally.

Proof. Using data in Table 5.1, we find that the coordinate $u$ always take distinct values on $P_{6}, P_{2}^{\prime}$, and $Q$ over $\mathbb{Z}[1 / 15]$. As a result, the isomorphism $u$, from $X_{\mathbb{Q}}^{D} / w_{3}$ to $\mathbb{P}_{\mathbb{Q}}^{1}$, extends to an isomorphism from $X_{\mathbb{Z}[1 / 15]}^{D} / w_{3} \rightarrow \mathbb{P}_{\mathbb{Z}[1 / 15]}^{1}$. 
The element $(\sqrt{-7}-3) / 6$ has minimal polynomial $x^{2}+x+4 / 9$, and hence is an algebraic integer over $\mathbb{Z}[1 / 15]$ (so it never coincides with $\infty$ in $\mathbb{P}^{1}$ over this ring). Moreover, substituting $u=0$ into Equation (4.1) we also find that

$$
\left(\frac{\sqrt{-7}-3}{6}-\omega\right)\left(\frac{\sqrt{-7}-3}{6}-\omega^{2}\right)=\frac{5}{9},
$$

so the element $(\sqrt{-7}-3) / 6-\omega$ is a unit over $\mathbb{Z}[1 / 15]$. This shows that $v$ also takes distinct values on $P_{6}, P_{2}^{\prime}$, and $Q$ over $\mathbb{Z}[1 / 15]$. Therefore, the map $v$ from $X_{\mathbb{Q}}^{D} / w_{15}$ to $\mathbb{P}_{\mathbb{Q}}^{1}$ extends to an isomorphism from $X_{\mathbb{Z}[1 / 15]}^{D} / w_{15} \rightarrow \mathbb{P}_{\mathbb{Z}[1 / 15]}^{1}$.

This demonstrates that Equation (4.1) gives a valid description, over $\mathbb{Z}[1 / 15]$, of $X^{D}$ as a closed subscheme of $\mathbb{P}^{1} \times \mathbb{P}^{1}$.

\section{Sections of the cotangent bundle}

Let $\kappa_{\mathcal{X}}$ be the relative cotangent bundle of $\mathcal{X}^{D}$, and $\kappa_{X}$ the (trivial) relative cotangent bundle of the coarse moduli scheme $X^{D}$.

As the map $\mathcal{X}^{D} \rightarrow X^{D}$ is triply ramified precisely over the degree-2 divisor $P_{6}$, there is an isomorphism

$$
H^{0}\left(\mathcal{X}^{D} ; \kappa_{\mathcal{X}}^{\otimes t}\right) \cong H^{0}\left(X^{D} ; \mathcal{O}\left(\lfloor 2 t / 3\rfloor P_{6}\right)\right) .
$$

Proposition 6.1. There is a section $a_{2} \in H^{0}\left(\mathcal{X}^{D} ; \kappa_{\mathcal{X}}\right)$ which has double zeros on the divisor $P_{6}$ and nowhere else.

Proof. There is a nowhere-vanishing 1-form on $X^{D}$, given by

$$
\frac{d u}{\left(u^{2}+u+1\right)(2 v+1)}=\frac{-d v}{\left(v^{2}+v+1\right)(2 u+1)} .
$$

This exhibits $\kappa_{X}$ as trivial. We denote the pullback of this to $\mathcal{X}^{D}$ by $a_{2}$. This section $a_{2}$ has a double pole along the divisor defining $P_{6}$ (which should be regarded as a $2 / 3$ pole). (Note that multiplication by $a_{2}^{n}$ induces the isomorphism (6.1).)

As in [HL10, 2.16], there is an identification of $\kappa_{\mathcal{X}}$ with the exterior square $\bigwedge^{2} \Omega$ of the relative cotangent bundle at the zero section of the universal abelian scheme $\mathcal{A} \rightarrow \mathcal{X}^{D}$.

We will choose an isomorphism $\Lambda \otimes \mathbb{Z}_{2} \cong M_{2}\left(\mathbb{Z}_{2}\right)$. This isomorphism determines, on $\mathcal{X}_{\mathbb{Z}_{2}}^{D}$, a splitting $\Omega \cong \omega \oplus \omega$, and thus an isomorphism of $\kappa_{\mathcal{X}}$ with $\omega^{2}$. 
COROLlaRY 6.2. There is a 2-torsion element in the Picard group of $\mathcal{X}_{\mathbb{Z}_{2}}^{D}$ which defines the obstruction to expressing $a_{2}$ as a unit times the square of a section $a_{1}$ of $\omega$.

Proof. The section $a_{2}$, with double zeros along the divisor $P_{6}$, defines an isomorphism of line bundles $\mathcal{O}\left(-2 P_{6}\right) \cong \kappa_{\mathcal{X}}$, or equivalently an isomorphism $\mathcal{O} \cong \kappa_{X}\left(2 P_{6}\right)$. The 2-torsion line bundle $\omega\left(P_{6}\right)$ then provides precisely this obstruction.

\section{Double covers}

In this section we will analyze double covers on $\mathcal{X}^{D}$. We begin with the following.

Proposition 7.1. For $p \in\{2,3,5\}$, there exists a nontrivial homomorphism $\sigma_{p}: \Lambda^{N=1} \rightarrow \mathbb{Z} / 2$ obtained by imposing level structure at the prime $p$. The effect on the generators of Corollary 2.2 is given in Table 7.1.

Proof. We first consider the ring map $\Lambda \rightarrow M_{2}(\mathbb{Z} / 2)$, given by

$$
\omega \mapsto\left[\begin{array}{ll}
0 & 1 \\
1 & 1
\end{array}\right], \quad y \mapsto\left[\begin{array}{ll}
0 & 1 \\
1 & 0
\end{array}\right] .
$$

In particular, $x=2 \omega+1$ maps to 1 . Under this map,

$$
h \mapsto\left[\begin{array}{ll}
0 & 1 \\
1 & 0
\end{array}\right], \quad \gamma \mapsto\left[\begin{array}{ll}
1 & 1 \\
1 & 0
\end{array}\right], \quad-1 \mapsto\left[\begin{array}{ll}
1 & 0 \\
0 & 1
\end{array}\right] .
$$

The composite homomorphism

$$
\sigma_{2}: \Lambda^{N=1} \rightarrow \mathrm{GL}_{2}(\mathbb{Z} / 2) \rightarrow\{ \pm 1\},
$$

which sends a matrix to the sign of its permutation action on $(\mathbb{Z} / 2)^{2}$, is then trivial on $\gamma$ and -1 and nontrivial on $h$.

We next have a ring homomorphism $\Lambda \rightarrow \mathbb{F}_{9}$ sending $\omega$ to 1 and $y$ to an element which is a square root of -1 , generating $\mathbb{F}_{9}$ over $\mathbb{F}_{3}$. Under this map,

$$
h \mapsto 1, \quad \gamma \mapsto \sqrt{-1}, \quad-1 \mapsto-1 .
$$

The composite map

$$
\sigma_{3}: \Lambda^{N=1} \rightarrow\left(\mathbb{F}_{9}\right)^{N=1} \cong \mathbb{Z} / 4 \rightarrow \mathbb{Z} / 2
$$

is trivial on $h$ and -1 , and nontrivial on $\gamma$. 
Table 7.1. Images of generators under double covers.

\begin{tabular}{cccc}
\hline Generator & $\sigma_{2}$ & $\sigma_{3}$ & $\sigma_{5}$ \\
\hline$h$ & 1 & 0 & 1 \\
$\gamma$ & 0 & 1 & 1 \\
-1 & 0 & 0 & 1 \\
\hline
\end{tabular}

Finally, we have a ring homomorphism $\Lambda \rightarrow \mathbb{F}_{25}$, sending $\omega$ to a third root of unity and $y$ to zero. Under this map,

$$
h \mapsto-1, \quad \gamma \mapsto-1, \quad-1 \mapsto-1
$$

The composite map

$$
\sigma_{5}: \Lambda^{N=1} \rightarrow\left(\mathbb{F}_{25}\right)^{N=1} \cong \mathbb{Z} / 6 \rightarrow \mathbb{Z} / 2
$$

is nontrivial on all three generators.

This shows that all elements of $H^{1}\left(\mathcal{X}_{\mathbb{C}}^{D} ; \mathbb{Z} / 2\right)$ come from some combination of these three algebraically defined level structures.

PROPOSITION 7.2. The character $\sigma_{3} \sigma_{5}$ induces a double cover $\mathcal{Y}_{\mathbb{C}} \rightarrow \mathcal{X}_{\mathbb{C}}^{D}$, obtained by imposing level structure away from the prime 2 , such that on $\mathcal{Y}_{\mathbb{C}}$ the form $a_{2}$ has a square root.

Proof. Using the embedding $\mathbb{Z}_{2} \rightarrow \mathbb{C}$, the section $a_{2}$ of $\omega^{2}$ is an automorphic form of weight 2 . We represent $a_{2}$ by a holomorphic function $a_{2}(z)$ on the upper half-plane $\mathcal{H}$, with double zeros at the elliptic points. It has a square root $a_{1}(z)$, which is almost an automorphic form of weight 1 . The group $\Lambda^{N=1}$ acts on $a_{1}$ by a character $\Lambda^{N=1} \rightarrow\{ \pm 1\}$.

The real structure $z \mapsto-\bar{z}$ is compatible with the action of $\Lambda^{N=1}$, and so every automorphic form $f(z)$ has a conjugate $\bar{f}(z)=\overline{f(-\bar{z})}$. As $a_{1}(z)$ and $\bar{a}_{1}(z)$ have the same zeros (concentrated at the elliptic points), they differ by a complex scalar; by rescaling we may assume that $a_{1}(z)$ takes real values on the imaginary axis.

We note that $(-1)$ must act nontrivially on $a_{1}$, as it acts by negation on all forms of weight 1 . Therefore, Table 7.1 tells us that the character of $\Lambda^{N=1}$ acting on $a_{1}$ is in the set $\left\{\sigma_{5}, \sigma_{2} \sigma_{5}, \sigma_{3} \sigma_{5}, \sigma_{2} \sigma_{3} \sigma_{5}\right\}$.

The element $h$ sends $a_{1}(z)$ to $a_{1}\left((4+\sqrt{15})^{2} z\right) /(4-\sqrt{15})$, which must be $\pm a_{1}(z)$. The function $t \mapsto a_{1}(i t)$ is an analytic function on the positive real line, 
taking real values, with zeros only at the points $(4+\sqrt{15})^{2 k} i$. In addition, these are simple zeros, which forces $a_{1}(i t)$ to change signs. Thus $h\left(a_{1}(z)\right)=-a_{1}(z)$.

By Table 7.1, this further reduces the possibilities for the character of $\Lambda^{N=1}$ acting on $a_{1}$ to the set $\left\{\sigma_{3} \sigma_{5}, \sigma_{5}\right\}$.

The Eichler-Selberg trace formula [Miy89] shows that the ring endomorphism associated to the Atkin-Lehner involution $w_{5}$ has trace 5 on the collection of forms of weight 2 , which is one dimensional and generated by $a_{2}(z)$. Therefore, $w_{5}$ must act on $a_{1}(z)$ by sending it to $\pm \sqrt{5} a_{1}(z)$. However, we have the identity $5 \gamma=\tilde{w}_{5}^{2}$ in $\Lambda$. This shows that $\gamma$ acts on $a_{1}(z)$ trivially, fixing the character as $\sigma_{3} \sigma_{5}$. This character can thus be obtained by imposing level structure away from the prime 2 .

Definition 7.3. Let $K$ denote the nontrivial unramified extension of $\mathbb{Q}_{2}$ of degree 4 , with ring of integers $\mathcal{O}_{K}=W\left(\mathbb{F}_{16}\right)$.

PROPOSITION 7.4. After choosing a complex embedding $K \rightarrow \mathbb{C}$, there is an étale double cover $\mathcal{Y} \rightarrow \mathcal{X}_{\mathcal{O}_{K}}^{D}$ over $\operatorname{Spec}\left(\mathcal{O}_{K}\right)$ inducing the double cover $\mathcal{Y}_{\mathbb{C}} \rightarrow \mathcal{X}_{\mathbb{C}}^{D}$. The composite $\mathcal{Y} \rightarrow \mathcal{X}_{\mathbb{Z}_{2}}^{D}$ is a Galois cover with Galois group $\mathbb{Z} / 8$.

Proof. The étale fundamental groups fit into the following diagram of groups with exact rows:

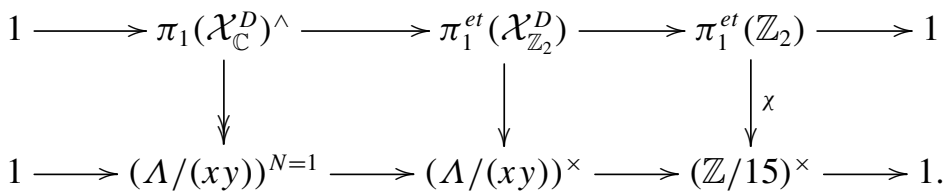

Here $\chi$ is the cyclotomic character, and the image of $\chi$ is generated by the element 2 - the image of the Frobenius automorphism. The images of the top row in the bottom row then fit into a commutative diagram as follows.

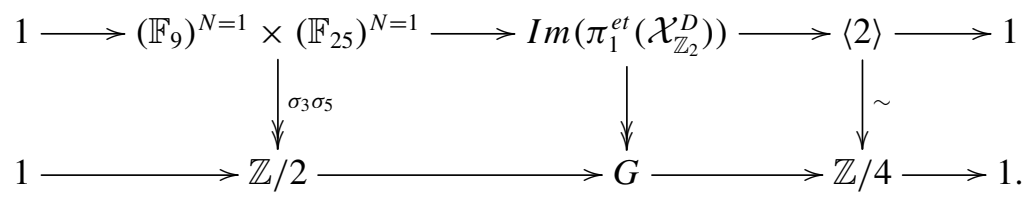

This explicitly determines an index-8 subgroup of $\pi_{1}^{e t}\left(\mathcal{X}_{\mathbb{Z}_{2}}^{D}\right)$ with quotient $G$, determining a composite degree- 8 cover

$$
\mathcal{Y} \rightarrow \mathcal{X}_{\mathcal{O}_{K}}^{D} \rightarrow \mathcal{X}_{\mathbb{Z}_{2}}^{D}
$$


Moreover, the pullback of $\mathcal{Y}$ along $\mathcal{X}_{\mathbb{C}}^{D} \rightarrow \mathcal{X}_{\mathcal{O}_{K}}^{D}$ is the double cover determined by the character $\sigma_{3} \sigma_{5}$, which is therefore $\mathcal{Y}_{\mathbb{C}}$.

We then verify directly (using the fact that $\mathbb{F}_{9}^{\times}$and $\mathbb{F}_{25}^{\times}$are cyclic) that the Frobenius element lifts to a generator of $G$, so that $G \cong \mathbb{Z} / 8$.

REMARK 7.5. As the element -1 has nontrivial image in $\{ \pm 1\}$ under the character $\sigma_{3} \sigma_{5}$, the double cover $\mathcal{Y}$ has the same geometric points as $\mathcal{X}_{\mathbb{Z}_{2}}^{D}$ and $\mathcal{X}_{\mathcal{O}_{K}}^{D}$.

REMARK 7.6. The recipe in this proof allows us to describe the stack $\mathcal{Y}$ as follows. There is a degree-192 cover $\mathcal{X}_{\mathbb{Z}_{2}}^{D}(\sqrt{15}) \rightarrow \mathcal{X}_{\mathbb{Z}_{2}}^{D}$ parameterizing twodimensional abelian schemes $A$ with $\Lambda$-action, together with a choice of primitive torsion point for $x y \in \Lambda$; the group $(\Lambda /(x y))^{\times}$is a Galois group for this cover, acting by the $\Lambda$-action on the torsion point. There are two components for the cover, each stabilized by $\operatorname{Im}\left(\pi_{1}^{e t}\left(\mathcal{X}_{\mathbb{Z}_{2}}^{D}\right)\right)$. The stabilizer group of one such component then contains an index-8 subgroup $H \subset(\Lambda /(x y))^{\times}$, and $\mathcal{Y}$ is the stack-theoretic quotient of this component by $H$.

Proposition 7.7. On the double cover $\mathcal{Y} \rightarrow \mathcal{X}_{\mathcal{O}_{K}}^{D}$ of Proposition 7.2, an $\mathcal{O}_{K^{-}}$ unit times the restriction of $a_{2}$ has a square root.

Proof. The étale fundamental group of $\mathcal{Y}_{K}$ fits into an exact sequence

$$
1 \rightarrow \pi_{1}\left(\mathcal{Y}_{\mathbb{C}}\right)^{\wedge} \rightarrow \pi_{1}^{e t}\left(\mathcal{Y}_{K}\right) \rightarrow \operatorname{Gal}\left(\overline{\mathbb{Q}}_{2} / K\right) \rightarrow 1
$$

which gives an exact sequence in cohomology

$$
0 \rightarrow K^{\times} /\left(K^{\times}\right)^{2} \rightarrow H^{1}\left(\mathcal{Y}_{K} ; \mathbb{Z} / 2\right) \rightarrow H^{1}\left(\mathcal{Y}_{\mathbb{C}} ; \mathbb{Z} / 2\right)
$$

There is an isomorphism $\mathbb{Z} / 2 \rightarrow \mu_{2}$ of étale sheaves on $\mathcal{Y}_{K}$, and so the étale cohomology group $\operatorname{Hom}\left(\pi_{1}^{e t}\left(\mathcal{Y}_{L}\right), \mathbb{Z} / 2\right)$ is the same as the étale cohomology group $H^{1}\left(\mathcal{Y}_{K} ; \mu_{2}\right)$ classifying 2 -torsion line bundles.

The 2-torsion line bundle $\omega\left(P_{6}\right)$ of Corollary 6.2, with chosen trivialization $a_{2}$ of its square, is classified by an element $\theta \in H^{1}\left(\mathcal{X}_{K}^{D} ; \mu_{2}\right)$. By Proposition 7.2, the image of $\theta$ in $H^{1}\left(\mathcal{Y}_{\mathbb{C}} ; \mathbb{Z} / 2\right)$ vanishes. The exact sequence (7.1) then shows that there is a form $a_{1}$ of weight 1 on $\mathcal{Y}_{K}$ such that $a_{1}^{2}=u a_{2}$ for a $K$-unit $u$.

Twice the divisor associated to $a_{1}$ is the pullback to $\mathcal{Y}$ of the divisor associated to $u a_{2}$. As $\mathcal{Y} \rightarrow \mathcal{X}_{\mathbb{Z}_{2}}^{D}$ is unramified over $2, u a_{2}$ can only have an even-degree zero or pole over the divisor (2) of $\mathcal{Y}$, and no other poles. We can therefore rescale $a_{1}$ by a multiple of 2 to lift it to a form defined on $\mathcal{Y}$ whose square is a $\mathcal{O}_{K}$-unit times $a_{2}$. 


\section{Automorphic forms on the cover}

Let $\mathcal{Y}$ be the double cover of $\mathcal{X}_{\mathcal{O}_{K}}^{D}$ from the previous section, with section $a_{1} \in H^{0}(\mathcal{Y} ; \omega)$ with simple zeros at the points lying over $P_{6}$. The coarse moduli scheme of $\mathcal{Y}$ is $X_{\mathcal{O}_{K}}^{D}$.

On $\mathcal{Y}$, the map taking a section $s$ to $s / a_{1}^{t}$ gives an identification of sections of $\omega^{\otimes t}$ with elements in the fraction field of $X_{\mathcal{O}_{K}}^{D}$ with poles of degree at most $\lfloor 2 t / 3\rfloor$ along the divisor $\{u=\infty\}$ defining $P_{6}$. In this section we will determine this graded ring.

Proposition 8.1. The graded ring

$$
\bigoplus_{t=0}^{\infty} H^{0}\left(X_{\mathcal{O}_{K}}^{D} ; \mathcal{O}\left(\lfloor 2 t / 3\rfloor P_{6}\right)\right)
$$

is isomorphic to a graded ring

$$
\mathcal{O}_{K}\left[a_{1}, a_{3}, a_{6}\right] / f\left(a_{i}\right) .
$$

Here $a_{1}$ has degree 1, $a_{3}$ has degree 3 , $a_{6}$ has degree 6 , and

$$
f\left(a_{i}\right)=a_{6}^{2}+a_{6}\left(a_{1}^{6}+a_{1}^{3} a_{3}+a_{3}^{2}\right)+\left(a_{1}^{6}+a_{1}^{3} a_{3}+a_{3}^{2}\right)^{2}-\frac{5}{9} a_{1}^{6}\left(a_{1}^{6}+a_{1}^{3} a_{3}+a_{3}^{2}\right) .
$$

Proof. From Equation (4.1), the fraction field of $X_{K}^{D}$ is

$$
K\left(X^{D}\right)=K(u)[v] /\left(u^{2}+u+1\right)\left(v^{2}+v+1\right)-\frac{5}{9} .
$$

A generic element of this field is $a(u)+b(u) v$. We find that this has no poles in the coordinate chart $u, v \neq \infty$ only when $a(u)$ and $b(u)$ are in $\mathcal{O}_{K}[u]$, and then has no poles in the coordinate chart $u \neq \infty, v \neq 0$ only when $\left(1+u+u^{2}\right)$ divides $b(u)$. Finally, the degree of the pole of $a(u)+b^{\prime}(u)\left(1+u+u^{2}\right) v$ over $P_{6}$ is the total degree in $u$.

As a consequence, the ring of Equation (8.1) is the graded ring generated by three elements, of weights 1,3 , and 6 :

$$
a_{1} \quad a_{3}=u a_{1}^{3} \quad a_{6}=\left(1+u+u^{2}\right) v a_{1}^{6} .
$$

The defining relation satisfied by $u$ and $v$, after multiplying by $a_{1}^{12}\left(1+u+u^{2}\right)$, becomes the minimal polynomial for $a_{6}$ :

$$
a_{6}^{2}+a_{6}\left(a_{1}^{6}+a_{1}^{3} a_{3}+a_{3}^{2}\right)+\left(a_{1}^{6}+a_{1}^{3} a_{3}+a_{3}^{2}\right)^{2}=\frac{5}{9} a_{1}^{6}\left(a_{1}^{6}+a_{1}^{3} a_{3}+a_{3}^{2}\right) .
$$

This gives a presentation of the ring of automorphic forms as desired. 
COROLLARY 8.2. The $\mathbb{Z} / 8$-action on the graded ring of automorphic forms $H^{0}\left(\mathcal{Y} ; \omega^{\otimes t}\right)$ is determined as follows. On the scalars $\mathcal{O}_{K}$ this action factors through the quotient $\mathbb{Z} / 8 \rightarrow \operatorname{Gal}\left(K / \mathbb{Q}_{2}\right)$, and the generator of $\mathbb{Z} / 8$ sends $a_{k}$ to $y^{k} \cdot a_{k}$ for an element $y \in \mathcal{O}_{K}$ with norm -1 which is congruent to 1 mod 2.

Proof. Since the group $H^{0}(\mathcal{Y}, \omega)$ is isomorphic to $\mathcal{O}_{K}$ with generator $a_{1}$, the generator $\sigma$ of $\mathbb{Z} / 8$ sends $a_{1}$ to $y \cdot a_{1}$ for some element $y \in \mathcal{O}_{K}^{\times}$. The element $\sigma^{4}$ acts on $a_{1}$ by negation, which is equivalent to $N_{K / \mathbb{Q}}(y)=-1$.

In $\mathbb{F}_{16}=\mathcal{O}_{K} /(2)$, Hilbert's theorem 90 implies that $y \equiv \sigma w / w$ for some unit $w$. By rescaling forms in degree $k$ by $w^{k}$, without loss of generality we may assume that $y$ reduces to one in $\mathbb{F}_{16}$.

As $a_{3}=u a_{1}^{3}$ and $a_{6}=\left(1+u+u^{2}\right) v a_{1}^{6}$ were obtained by multiplying $a_{1}^{k}$ by $\operatorname{Gal}\left(K / \mathbb{Q}_{2}\right)$-invariant rational functions on $X_{\mathbb{Q}_{2}}^{D}$, we obtain the desired action on the remainder of the ring.

\section{Cohomology of $\mathcal{X}_{\mathbb{Z}_{2}}^{D}$}

In this section we will exploit the ring of automorphic forms from Corollary 8.2 to determine the cohomology of $\mathcal{X}_{\mathbb{Z}_{2}}^{D}$ with coefficients in the tensor powers of $\omega$.

We first note that $\mathcal{Y}$ satisfies a type of Serre duality.

PROPOSITION 9.1. The cup product creates a perfect pairing

$$
H^{s}\left(\mathcal{Y} ; \omega^{\otimes t}\right) \otimes H^{1-s}\left(\mathcal{Y} ; \omega^{\otimes(2-t)}\right) \rightarrow H^{1}\left(\mathcal{Y} ; \omega^{\otimes 2}\right) \cong \mathcal{O}_{K} .
$$

Proof. The stack $\mathcal{Y}$ has a cover by the open substacks $a_{1}^{-1} \mathcal{Y}$ and $a_{3}^{-1} \mathcal{Y}$, each of which has an affine scheme as its coarse moduli object and whose higher cohomology vanishes because all points have automorphism groups of odd order. In terms of the graded ring of Corollary 8.2, the Mayer-Vietoris sequence then degenerates to an exact sequence

$$
0 \rightarrow R_{*} \rightarrow a_{1}^{-1} R_{*} \oplus a_{3}^{-1} R_{*} \rightarrow\left(a_{1} a_{3}\right)^{-1} R_{*} \rightarrow H^{1}\left(\mathcal{Y} ; \omega^{\otimes *}\right) \rightarrow 0 .
$$

In particular, the element $D=\left(a_{1} a_{3}\right)^{-1} a_{6}$ maps to a generating element in $H^{1}$, which makes the monomial basis $a_{1}^{k} a_{3}^{l} a_{6}^{\epsilon}$ of $H^{0}$ dual to the basis elements which are the image of $\left(a_{1}\right)^{-1-k}\left(a_{3}\right)^{-1-l}\left(a_{6}\right)^{1-\epsilon}$.

We now employ the cohomology spectral sequence

$$
H^{p}\left(\mathbb{Z} / 8 ; H^{q}\left(\mathcal{Y} ; \omega^{\otimes t}\right)\right) \Rightarrow H^{p+q}\left(\mathcal{X}_{\mathbb{Z}_{2}}^{D} ; \omega^{\otimes t}\right) .
$$

This spectral sequence is concentrated either in $q=0$ or $q=1$, according to the value of $t$. Therefore, this collapses to the group cohomology, with terms having degrees shifted according to whether they originate in $H^{0}(\mathcal{Y})$ or $H^{1}(\mathcal{Y})$. 
THEOREM 9.2. Consider the bigraded ring

$$
H^{s}\left(\mathcal{Y} ; \omega^{\otimes t}\right)[\zeta] /(2 \zeta)
$$

where $\zeta$ is in degree $(s, t)=(1,0)$. This ring has an additional $\mathbb{Z} / 2$-grading: $a_{1}$, $a_{3}$, and $\zeta$ have odd grading, while $a_{6}$ and $D$ have even grading.

The cohomology of $\mathbb{Z} / 2 \subset \mathbb{Z} / 8$ with coefficients in $H^{s}\left(\mathcal{Y} ; \omega^{\otimes t}\right)$ is the subgroup of elements in bidegree $(s, t)$ of even grading, and the cohomology of $\mathbb{Z} / 8$ with with coefficients in $H^{s}\left(\mathcal{Y} ; \omega^{\otimes t}\right)$ consists of those elements invariant under the map $x \mapsto y^{t} \sigma(x)$, where $y$ is the element of norm -1 from Corollary 8.2 and $\sigma$ is a generator of the Galois group.

Proof. The calculation of the cohomology of $\mathbb{Z} / 2$ is standard. In addition, because the extension $\mathbb{Z}_{2} \rightarrow \mathcal{O}_{K}$ is unramified the terms in the spectral sequence

$$
H^{p}\left(\mathbb{Z} / 4 ; H^{q}\left(\mathbb{Z} / 2 ; H^{*}\left(\mathcal{Y} ; \omega^{\otimes t}\right)\right)\right) \Rightarrow H^{p+q}\left(\mathbb{Z} / 8 ; H^{*}\left(\mathcal{Y} ; \omega^{\otimes t}\right)\right)
$$

vanish for $p>0$. Therefore,

$$
H^{q}\left(\mathbb{Z} / 8 ; H^{*}\left(\mathcal{Y} ; \omega^{\otimes t}\right)\right) \cong H^{q}\left(\mathbb{Z} / 2 ; H^{*}\left(\mathcal{Y} ; \omega^{\otimes t}\right)\right)^{\mathrm{Gal}\left(K / \mathbb{Q}_{2}\right)},
$$

as desired.

As $y$ is congruent to $1 \bmod 2$, elements involving a positive power of $\zeta$ are invariant under the action of $\operatorname{Gal}\left(K / \mathbb{Q}_{2}\right)$ if and only if their coefficients come from $\mathbb{F}_{2} \subset \mathbb{F}_{16}$.

As $y^{2}$ has norm 1, Hilbert's theorem 90 implies that there exists an element $x \in \mathcal{O}_{K}^{\times}$with $y^{2}=x / \sigma(x)$; the element $x$ must also reduce to 1 in $\mathbb{F}_{16}$. We formally define $b_{i}=x^{i / 2} a_{i}$.

Then the elements $b_{i}$ generate a ring $\mathbb{Z}_{2}\left[b_{1}, b_{3}, b_{6}\right] /\left(f\left(b_{i}\right)\right)$. The ring $H^{0}\left(\mathbb{Z} / 8 ; H^{0}\left(\mathcal{Y} ; \omega^{\otimes t}\right)\right)$ is the subring consisting of those elements in even total weight. Similarly, the module $H^{0}\left(\mathbb{Z} / 8 ; H^{1}\left(\mathcal{Y} ; \omega^{\otimes t}\right)\right)$ is, as a consequence of the Mayer-Vietoris sequence, generated by elements of the form $\left(b_{1}\right)^{-1-j}\left(b_{3}\right)^{-1-k} b_{6}^{1-\epsilon}$ in even total weight.

\section{The Atkin-Lehner involution}

The following is an expansion on what appears in [HL10, 5.6].

Proposition 10.1. The 2-divisible group on $\mathcal{X}^{D}$ descends to one on the quotient stack $\mathcal{X}^{D} / w_{15}$. 
Proof. We first recall the definition of the quotient stack.

For a scheme $Y$, the $Y$-points $\mathcal{X}^{D}(Y)$ form the category of two-dimensional abelian schemes $A$ over $Y$ with an action of $\Lambda$, and morphisms are $\Lambda$-linear isomorphisms. The endofunctor $w_{15}$ sends $A$ to $w_{15}(A)$, which has the same underlying abelian scheme; the action is precomposed with the automorphism of $\Lambda$ given by conjugation by $x y \in D$. (Note that the $\Lambda$-linear map $x y: A \rightarrow w_{15}(A)$ factors through an isomorphism $A / A[x y] \rightarrow w_{15}(A)$, between $w_{15}(A)$ and the quotient of $A / A[x y]$ by the subgroup of points annihilated by $x y$.) We have $\left(w_{15}\right)^{2}=I d$. The stack $\mathcal{X}^{D} / w_{15}$ is obtained by stackifying the functor whose $Y$ points form a groupoid with the same objects as those of $\mathcal{X}^{D}$; the set of morphisms from $A$ to $B$ is the disjoint union of the set of isomorphisms $A \rightarrow B$ and the set of isomorphisms $w_{15} A \rightarrow B$.

We choose a 2-adic square root of -15 . The 2-divisible group of $\mathcal{X}^{D}$ takes a $Y$-point $A$ to the 2-divisible group $A\left[2^{\infty}\right]$. To extend this 2-divisible group to $\mathcal{X}^{D} / w_{15}$, for an isomorphism $w_{15} A \rightarrow B$ we assign $1 / \sqrt{-15}$ times the composite isomorphism

$$
A\left[2^{\infty}\right] \stackrel{x y}{\longrightarrow}\left(w_{15} A\right)\left[2^{\infty}\right] \rightarrow B\left[2^{\infty}\right]
$$

To verify that this preserves composition, we must check that it commutes with $w_{15}$, which is straightforward.

PROPOSITION 10.2. The involution on the ring of automorphic forms induced by $w_{15}$ acts trivially on the generators $b_{1}^{2}, b_{1} b_{3}$, and $b_{3}^{2}$, and sends $b_{6}$ to $-b_{6}-\left(b_{1}^{6}+\right.$ $\left.b_{1}^{3} b_{3}+b_{3}^{2}\right)$.

Proof. We can apply the Eichler-Selberg trace formula (as in [HL10, Section 3.5]) to determine the action of the Atkin-Lehner operators on the ring of automorphic forms. It shows that the ring endomorphism associated to $w_{15}$ has trace -15 in degree 2 , and $2(-15)^{k}$ in degrees $2 k>2$.

The induced involution which gives rise to the 2-divisible group on the quotient stack $\mathcal{X}^{D} / w_{15}$ rescales the action on forms in weight $k$ by $(\sqrt{-15})^{-k}$; it therefore has trace 1 in degree 2 and trace 2 in higher degrees.

The form $b_{1}^{2}$ generates all forms of weight 2 , and is thus fixed; the forms $b_{1}^{4}$ and $b_{1} b_{3}$ generate all forms of weight 4 , and are fixed as well. Since $b_{3}^{2} b_{1}^{2}=\left(b_{1} b_{3}\right)^{2}$ and $b_{1}^{2}$ is not a zero divisor, $b_{3}^{2}$ must also be fixed.

As the forms $b_{1}^{6}, b_{3} b_{1}^{3}$, and $b_{3}^{2}$ are all fixed, the remaining generator $b_{6}$ in degree 6 must be sent to $-b_{6}$ plus a fixed element. The result must satisfy the same minimal polynomial as $b_{6}$, so it is forced to map to the conjugate root $-b_{6}-$ $\left(b_{1}^{6}+b_{1}^{3} b_{3}+b_{3}^{2}\right)$, as desired. 
PROPOSITION 10.3. The involution $w_{15}$ lifts to an involution on the double cover $\mathcal{Y}$, along with its 2-divisible group, that fixes the forms $a_{1}$ and $a_{3}$ and commutes with the $\mathbb{Z} / 2$-action on $\mathcal{Y}$.

Proof. By Remark 7.6, the points of $\mathcal{Y}$ are locally described as abelian schemes $A$ equipped with an equivalence class $[p]$ of $x y$-torsion point under the action of a subgroup of $H<(\Lambda /(x y))^{\times}$. Conjugation by $x y$ preserves this subgroup, and so we can lift the involution $w_{15}$ to an involution $(A,[p]) \mapsto\left(w_{15} A,[p]\right)$ of $\mathcal{Y}$. This is an automorphism over $\mathcal{O}_{K}$ and it commutes with the $\mathbb{Z} / 2$-action, which sends $[p]$ to $[k p]$ for some $k \in \Lambda$.

This lift of $w_{15}$ fixes $b_{1}^{2}=x a_{1}^{2}$, and so must either fix $a_{1}$ or negate it; however, by possibly composing with the $\mathbb{Z} / 2$-action on $\mathcal{Y}$ we can replace it with a lift fixing $a_{1}$. It then fixes $a_{1}$ and $a_{1} a_{3}$, and since $a_{1}$ is not a zero divisor it fixes $a_{3}$.

\section{Spectra}

As in [HL10, 2.6], the 2-divisible group on the Deligne-Mumford stack $\mathcal{X}^{D} / w_{15}$ gives rise to a sheaf $\mathcal{O}^{\text {der }}$ of spectra on the étale site of $\left(\mathcal{X}^{D} / w_{15}\right)_{2}^{\wedge}$. In particular, we can define global section objects:

$$
\begin{aligned}
E & =\Gamma\left(\mathcal{Y}_{2}^{\wedge}, \mathcal{O}^{d e r}\right) \\
\operatorname{TAF}^{D} & =\Gamma\left(\left(\mathcal{X}^{D}\right)_{2}^{\wedge}, \mathcal{O}^{d e r}\right) \\
E^{w_{15}} & =\Gamma\left(\left(\mathcal{Y} / w_{15}\right)_{2}^{\wedge}, \mathcal{O}^{d e r}\right) \\
\left(\mathrm{TAF}^{D}\right)^{w_{15}} & =\Gamma\left(\left(\mathcal{X}^{D} / w_{15}\right)_{2}^{\wedge}, \mathcal{O}^{\text {der }}\right) .
\end{aligned}
$$

The expressions of $\mathcal{X}^{D}, \mathcal{Y} / w_{15}$, and $\mathcal{X}^{D} / w_{15}$ as quotients of $\mathcal{Y}$ by group actions give us fixed-point expressions:

$$
\begin{aligned}
\mathrm{TAF}^{D} & \simeq E^{h(\mathbb{Z} / 8 \times 1)} \\
E^{w_{15}} & \simeq E^{h(1 \times \mathbb{Z} / 2)} \\
\left(\mathrm{TAF}^{D}\right)^{w_{15}} & \simeq E^{h(\mathbb{Z} / 8 \times \mathbb{Z} / 2)} .
\end{aligned}
$$

Our goal in this section is to analyze the resulting homotopy fixed-point spectral sequences. We note that the $\mathbb{Z} / 8 \times \mathbb{Z} / 2$-invariant cover of $\mathcal{Y}$ by $a_{1}^{-1} \mathcal{Y}$ and $a_{3}^{-1} \mathcal{Y}$ gives rise to an equivariant homotopy pullback diagram:

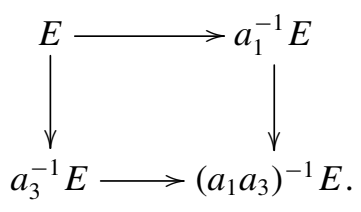


On homotopy groups, this determines a square which is part of a Mayer-Vietoris sequence:

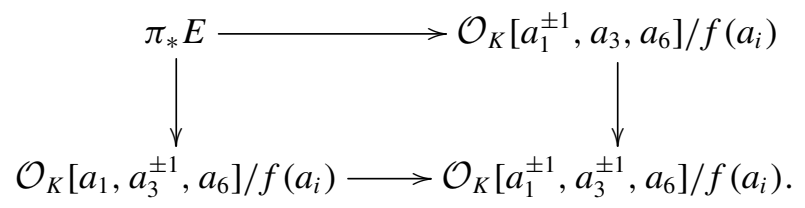

Proposition 11.1. There is an $E_{\infty}$ ring spectrum $e$ with a $\mathbb{Z} / 8 \times \mathbb{Z} / 2$ equivariant map $e \rightarrow E$ which, on homotopy groups, is the inclusion

$$
\mathcal{O}_{K}\left[a_{1}, a_{3}, a_{6}\right] / f\left(a_{i}\right) \rightarrow \pi_{*} E
$$

of terms in even total degree which were contributed by $H^{0}(\mathcal{Y})$.

Proof. Let $\tilde{e}$ be the connective cover of $E$; the map $\tilde{e} \rightarrow E$ is a $\mathbb{Z} / 8 \times \mathbb{Z} / 2$ equivariant map, and it has the desired behavior on homotopy groups except that $\pi_{1} \tilde{e}$ is $\mathcal{O}_{K} \neq 0$. Let $P_{1} \tilde{e}=\tilde{e}[0,1]$ be the Postnikov stage of $\tilde{e}$. As the element $\eta \in$ $\pi_{1}\left(\mathbb{S}_{2}^{\wedge}\right)$ maps to zero in $\pi_{1}(\tilde{e})$, the map $\mathbb{S}_{2}^{\wedge} \rightarrow P_{1} \tilde{e}$ factors through the Postnikov stage of the equivariant $E_{\infty}$ cone on $\eta$, which is $H \mathbb{Z}_{2}$. Similarly, attaching cells to $H \mathbb{Z}_{2}$ allows us to extend to a map of $E_{\infty}$ rings $H \mathcal{O}_{K} \rightarrow P_{1} e$. We can therefore form the homotopy pullback

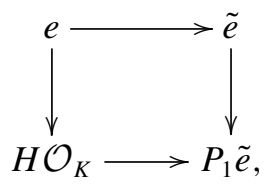

which has the desired properties.

A direct consideration of homotopy groups gives the following.

COROLlary 11.2. The maps $a_{1}^{-1} e \rightarrow a_{1}^{-1} E$ and $a_{3}^{-1} e \rightarrow a_{3}^{-1} E$ are equivalences.

\section{Homotopy groups of $\mathbf{T A F}^{D}$}

12.1. Connective fixed points. We begin to compute the homotopy groups of $\mathrm{TAF}^{D}$ by analyzing the homotopy fixed-point spectral sequence for the spectrum $e$ in Proposition 11.1. These carry a very strong resemblance to the calculations of [MR09], with the addition of the generator $b_{6}$ which operates largely independently.

We will write $\zeta$ for the generating element of $H^{1}\left(\mathbb{Z} / 2 ; \mathbb{Z}_{2}^{s g n}\right)$. This allows us to give the following short expression of the group cohomology. 
PROPOSITION 12.1. The $E_{2}$-term of the homotopy fixed-point spectral sequence for $\mathbb{Z} / 8$ acting on e takes the following form:

$$
E_{2}^{s, t}=\mathbb{Z}_{2}\left[b_{1}^{2}, b_{1} b_{3}, b_{3}^{2}, b_{6}, \zeta^{2}, \zeta a_{1}, \zeta a_{3}\right] /\left(2 \zeta, f\left(b_{i}\right)\right) .
$$

In particular, these terms are concentrated in degrees with $(t-s) \equiv s \bmod 4$, and the only possible differentials are $d_{4 k+3}$.

REMARK 12.2. As $x$ reduces to $1 \bmod 2, \zeta x=0$, and hence for any polynomial $g\left(a_{1}, a_{3}, a_{6}\right)$ which is homogeneous of even degree we have $\zeta g\left(a_{1}, a_{3}, a_{6}\right)=$ $\zeta g\left(b_{1}, b_{3}, b_{6}\right)$. This allows us to abuse notation by writing $\zeta b_{i}=\zeta a_{i}$.

Proposition 12.3. Under the map $\pi_{*} \mathbb{S} \rightarrow e^{h \mathbb{Z} / 2}$, the images of $\eta \in \pi_{1} \mathbb{S}$ and $v \in \pi_{3} \mathbb{S}$ are represented by $\zeta b_{1}$ and $\zeta^{3} b_{3}$ in the homotopy fixed-point spectral sequence.

Proof. Completion at the supersingular locus gives a map $e \rightarrow E_{2}$ to a LubinTate spectrum over $\mathbb{F}_{16}$, which is expressed on homotopy groups

$$
\mathbb{Z}_{2}\left[b_{1}, b_{3}, b_{6}\right] / f\left(b_{i}\right) \rightarrow \mathcal{O}_{K} \llbracket u_{1} \rrbracket\left[u^{ \pm 1}\right]
$$

by sending $b_{1}$ to $u_{1} u, b_{3}$ to $u^{3}$, and $b_{6}$ to the unique root of $f\left(b_{i}\right)$ which reduces, $\bmod \left(2, u_{1}\right)$, to $\omega u^{6}$, where $\omega$ is a chosen primitive third root of unity. This expresses $L_{K(2)} e$ as a homotopy fixed-point spectrum of $E_{2}$ by an action of $\mathbb{Z} / 3$, and the $\mathbb{Z} / 2$-action on $e$ realizes to the action of $[-1]$ as a subgroup of the Morava stabilizer. The elements $\eta$ and $v$ are detected on the 1-line and 3-line respectively of the associated homotopy fixed-point spectral sequence for $E_{2}$, by a transfer argument in the latter case [MR09]. They are hence also detected in the homotopy fixed-point spectral sequence for $e$.

PROPOSITION 12.4. In the homotopy fixed-point spectral sequence, we have differentials

$$
d_{3}\left(\zeta^{2}\right)=\zeta^{5} b_{1} \quad \text { and } \quad d_{7}\left(\zeta^{4}\right)=\zeta^{11} b_{3}
$$

Proof. In the homotopy fixed-point spectral sequence for the action of $\mathbb{Z} / 2$ on $\mathbb{S}$, which coincides with the Atiyah-Hirzebruch spectral sequence for the stable cohomotopy of $\mathbb{R} \mathbb{P}^{\infty}$, the cell attachment structure of $\mathbb{R} \mathbb{P}^{\infty}$ implies that we have differentials

$$
d_{2}\left(\zeta^{2}\right)=\zeta^{4} \eta, \quad \text { and } \quad d_{4}\left(\zeta^{4}\right)=\zeta^{8} v
$$


We truncate to a skeleton of $E \mathbb{Z} / 2$ and compare this with the homotopy fixedpoint spectral sequence for $e$ :

$$
F\left(\mathbb{R P} \mathbb{P}^{k}, \mathbb{S}\right) \rightarrow F_{\mathbb{Z} / 2}\left((E \mathbb{Z} / 2)^{(k)}, e\right) .
$$

When $k=5$, the differentials we described show that $\zeta^{4} \eta$ is either trivial or detected by $\zeta^{5} \eta^{2}$ in the spectral sequence calculating $\pi_{-4} F\left(\mathbb{R} \mathbb{P}^{5}, \mathbb{S}\right)$. Therefore, $\zeta^{5} b_{1}$ is equivalent to a class which could only be detected by 0 or the class $\zeta^{7} b_{1}^{2}=0$ in the spectral sequence calculating $\pi_{*} F_{\mathbb{Z} / 2}\left((E \mathbb{Z} / 2)^{(5)}, e\right)$, and thus must be the target of a differential; the only possible source is $\zeta^{2}$.

Similarly, when $k=11$ the differentials show that $\zeta^{8} v$ is either trivial or detected by $\zeta^{11} v$; in the spectral sequence calculating $\pi_{*} F_{\mathbb{Z} / 2}\left((E \mathbb{Z} / 2)^{(5)}, e\right)$ the element $\zeta^{11} b_{3}$ is equivalent to a class that could only be detected by zero or $\zeta^{14} b_{3}=0$. It then must be the target of a differential; the only possible source after the $E_{2}$-page is $\zeta^{4}$.

Proposition 12.5. The $d_{3}$-differentials in the homotopy fixed-point spectral sequence are determined by the following differentials and the Leibniz rule:

$$
\begin{aligned}
& d_{3}\left(\zeta^{2}\right)=\zeta^{5} b_{1} \quad d_{3}\left(b_{1}^{2}\right)=\zeta^{3} b_{1}^{3} \quad d_{3}\left(b_{3}^{2}\right)=\zeta^{3} b_{1} b_{3}^{2} \quad d_{3}\left(b_{1} b_{3}\right)=0 \\
& d_{3}\left(\zeta b_{1}\right)=0 \quad d_{3}\left(\zeta b_{3}\right)=\zeta^{4} b_{1} b_{3} \quad d_{3}\left(b_{6}\right)=\zeta^{3} b_{1} b_{6} .
\end{aligned}
$$

Proof. The differential on $\zeta^{2}$ is determined by the previous proposition.

For a class in even total degree which is negated by the $\mathbb{Z} / 2$-action, [MR09] describes a cup-1 identity $d_{3}\left(x^{2}\right)=\eta(\zeta x)^{2}$ in the homotopy fixed-point spectral sequence for the action of the subgroup $\mathbb{Z} / 2$, which determines a nontrivial differential

$$
d_{3}\left(b_{1}^{2}\right)=d_{3}\left(x a_{1}^{2}\right)=x \zeta^{3} b_{1}^{3}=\zeta^{3} b_{1}^{3} .
$$

Naturality of the spectral sequence implies that there is a corresponding differential in the homotopy fixed-point spectral sequence for the action of $\mathbb{Z} / 8$. The differential on $b_{3}^{2}$ follows similarly.

The elements $\zeta b_{1}$ and $\zeta^{3} b_{3}$ support no differentials by Proposition 12.3.

The Leibniz rule shows the identity

$$
0=d_{3}\left(\left(\zeta b_{1}\right)\left(\zeta^{3} b_{3}\right)\right)=\zeta^{4} d_{3}\left(b_{1} b_{3}\right) .
$$

Similarly, we have

$$
0=d_{3}\left(\zeta^{3} b_{3}\right)=\zeta^{6} b_{1} b_{3}+\zeta^{2} d_{3}\left(\zeta b_{3}\right) .
$$

Multiplication by $\zeta^{2}$ induce isomorphisms from the $s$-line to the $s+2$-line for $s>0$, and so this forces the identities $d_{3}\left(b_{1} b_{3}\right)=0$ and $d_{3}\left(\zeta b_{3}\right)=\zeta^{4} b_{1} b_{3}$. 


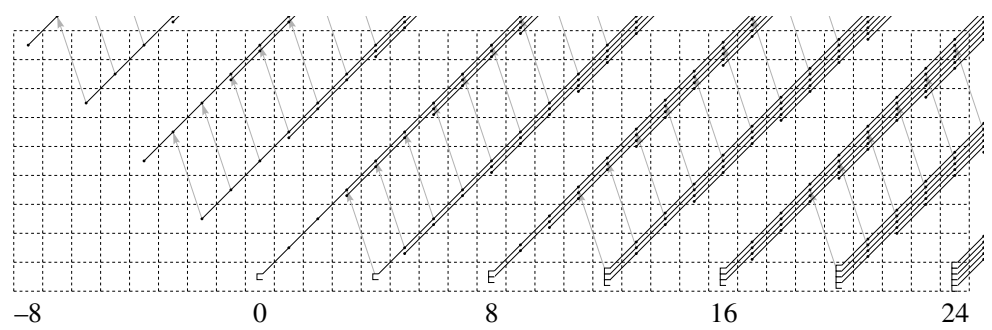

Figure 12.1. $E_{3}$-page, classes which are not multiples of $b_{6}$.

Finally, applying $d_{3}$ to the identity $f\left(b_{i}\right)=0$ gives rise to the identity

$$
0=d_{3}\left(b_{6}\right)\left(b_{1}^{6}+b_{1}^{3} b_{3}+b_{3}^{2}\right)+\left(b_{1}^{6}+b_{1}^{3} b_{3}+b_{3}^{2}\right) \zeta^{3} b_{1} b_{6},
$$

which forces the desired differential on $b_{6}$.

It is convenient to write a class on the zero-line in the form $b_{1}^{4 k-3 l} b_{3}^{l}$ or $b_{1}^{2+4 k-3 l} b_{3}^{l} b_{6}$ if it is in degree congruent to $0 \bmod 8$, and in the form $b_{1}^{-2+4 k-3 l} b_{3}^{l}$ or $b_{1}^{4 k-3 l} b_{3}^{l} b_{6}$ if it is in degree equivalent to $4 \bmod 8$. In these terms we have

$$
\begin{aligned}
d_{3}\left(b_{1}^{2+4 k-3 l} b_{3}^{l}\right) & =\eta^{3} \cdot b_{1}^{4 k-3 l} b_{3}^{l}, \\
d_{3}\left(b_{1}^{4(k+1)-3 l} b_{3}^{l} b_{6}\right) & =\eta^{3} \cdot b_{1}^{2+4 k-3 l} b_{3}^{l} b_{6} .
\end{aligned}
$$

More, we have differentials

$$
d_{3}\left(\zeta^{4 k+2}\right)=\eta \cdot \zeta^{4 k+4} \quad \text { and } \quad d_{3}\left(\zeta^{4 k} b_{6}\right)=\eta \cdot \zeta^{4 k+2} b_{6} .
$$

Mod 2, we then find that $d_{3}$ is injective on classes where $t-2 s \equiv 4 \bmod 8$.

A schematic diagram of this differential appears in Figures 12.1 and 12.2, broken up according to whether the classes are multiples of $b_{6}$. Note that an arrow indicates that all classes in the indicated degree support a differential.

The surviving classes on the zero-line are generated by

$$
b_{1}^{4}, b_{1} b_{3}, b_{3}^{4}, b_{1}^{2} b_{6}, b_{3}^{2} b_{6}, 2 b_{1}^{2}, 2 b_{3}^{2}, 2 b_{6} ;
$$

the classes which are not multiples of 2 support $\eta^{2}$-multiples. In filtrations above 2 , almost all classes are annihilated. The remaining ones are all $\nu$-multiples of the classes $\eta^{k} \zeta^{4 l}$ and $\eta^{k} \zeta^{4 l+2} b_{6}$ for $0 \leqslant k \leqslant 2$, $\zeta^{l} b_{3}^{4 k-l}$, and $\zeta^{l} b_{3}^{4 k-l+2} b_{6}$. The remaining classes on the 1-line and 2-line consist only of the $b_{3}^{4}$-multiples of the following classes:

$$
\zeta^{2} b_{3}^{2}, \zeta b_{3}^{3}, \eta \zeta b_{3}^{3}, \zeta b_{3} b_{6}, \zeta^{2} b_{6}, \eta \zeta b_{3} b_{6} .
$$




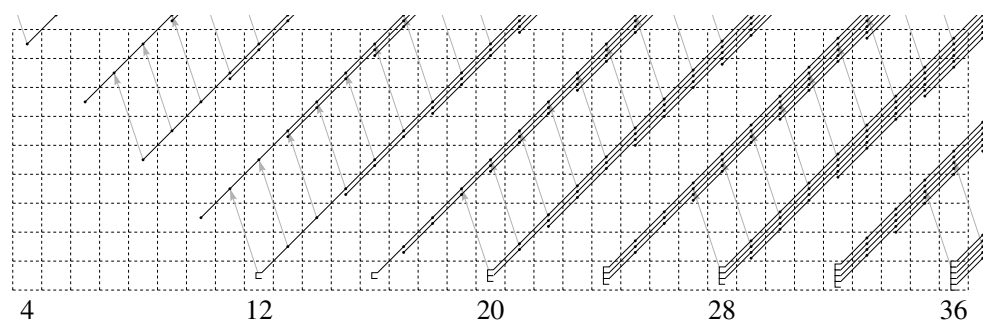

Figure 12.2. $E_{3}$-page, classes which are multiples of $b_{6}$.

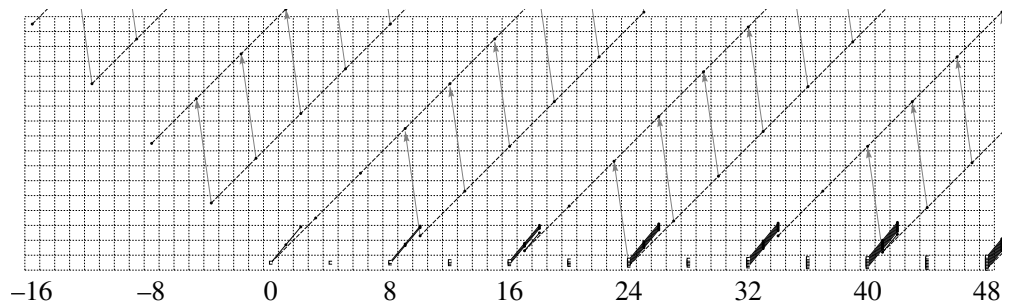

Figure 12.3. $E_{7}$-page, classes which are not multiples of $b_{6}$.

Multiplications by $b_{3}^{4}, \zeta^{4}$, and $v$ are injective in filtrations greater than or equal to 3 .

PROPOSITION 12.6. The only nonzero $d_{7}$-differentials in the homotopy fixedpoint spectral sequence are

$$
\begin{array}{rlll}
d_{7}\left(\zeta^{k} b_{3}^{l}\right)=\zeta^{k+7} b_{3}^{l+2} & \text { if } 3 l-k \equiv 4 \quad \bmod 8, \quad \text { and } \\
d_{7}\left(\zeta^{k} b_{3}^{l} b_{6}\right)=\zeta^{k+7} b_{3}^{l+2} b_{6} & \text { if } 3 l-k \equiv 6 \quad \bmod 8 .
\end{array}
$$

This differential is pictured in Figures 12.3 and 12.4; dashed lines indicate multiplication by $v$. It may be more concisely expressed as saying that $\nu$-towers in degrees $t-2 s \equiv 8 \bmod 16$ support differentials which hit the $v$-towers in degrees $t-2 s \equiv 0 \bmod 16$.

Proof. We first note that all possible targets of a differential are elements which are not $\nu$-torsion, and so the only possible sources of a differential are the non- $v$ torsion elements $\zeta^{k} b_{3}^{l}$ and $\zeta^{k} b_{3}^{l} b_{6}$ which have survived to $E_{7}$.

We have differentials $d_{7} \zeta^{4}=v \zeta^{8}$, and $d_{7} v=0$; by the Leibniz rule, we then find the desired differentials on all classes of the form $\zeta^{k} b_{3}^{l}$. 


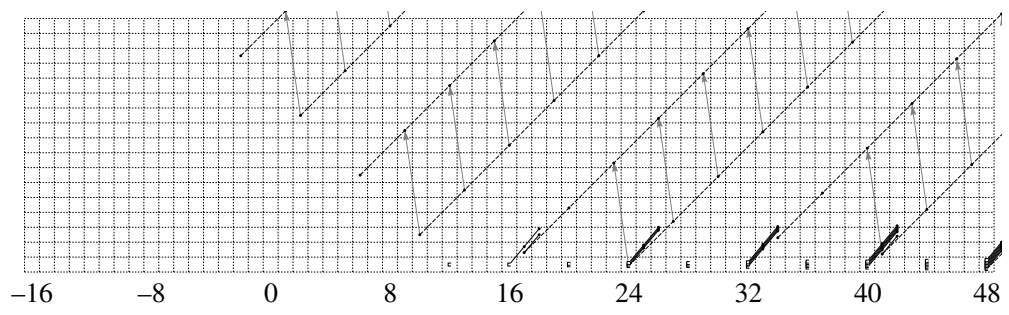

Figure 12.4. $E_{7}$-page, classes which are multiples of $b_{6}$.

Multiplying the equation $f\left(b_{i}\right)=0$ by $\zeta^{4}$ gives the identity on $E_{7}$-pages:

$$
\left(\zeta^{2} b_{6}\right)^{2}+\left(\zeta^{2} b_{3}^{2}\right)\left(\zeta^{2} b_{6}\right)+\left(\zeta^{2} b_{3}^{2}\right)^{2}=0 .
$$

The Leibniz rule then implies $d_{7}\left(\zeta^{2} b_{3}^{2}\right)\left(\zeta^{2} b_{6}\right)=\zeta^{2} b_{3}^{2} d_{7}\left(\zeta^{2} b_{6}\right)$, and thus $d_{7}\left(\zeta^{2} b_{6}\right)=v \zeta^{4}\left(\zeta^{2} b_{6}\right)$. The Leibniz rule then determines the differentials on all elements $\zeta^{k} b_{3}^{l} b_{6}$.

There is no room for further differentials; the only remaining classes in high filtration are $\zeta^{8 k}$ and $\zeta^{8 k+6} b_{6}$, which are in even total degree and cannot be a target of a differential.

The following assembles the final result.

THEOREM 12.7. The nonnegative-degree homotopy groups of $e^{\text {h } / 2}$ fit into a short exact sequence $0 \rightarrow K \rightarrow \pi_{*} e^{h \mathbb{Z} / 2}\langle 0\rangle \rightarrow R \rightarrow 0$. The terms in this sequence are given as follows.

- $R$ is the pullback in the diagram of rings

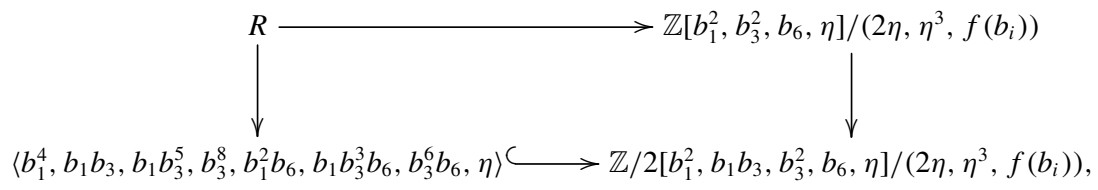

where the lower map is the inclusion of the subring generated by the given elements.

- $K$ is freely generated over $\mathbb{Z} / 2\left[b_{3}^{8}\right]$ by the following classes:

$$
\begin{array}{ll}
\nu, v^{2}, & v\left(b_{3}^{6} b_{6}\right), v^{2}\left(b_{3}^{6} b_{6}\right), \\
\zeta b_{3}^{3}, \eta\left(\zeta b_{3}^{3}\right), v\left(\zeta b_{3}^{3}\right), & \zeta b_{3} b_{6}, \eta\left(\zeta b_{3} b_{6}\right), v\left(\zeta b_{3} b_{6}\right), \\
\eta\left(b_{3}^{4}\right), \eta^{2}\left(b_{3}^{4}\right), & \eta\left(b_{3}^{2} b_{6}\right), \eta^{2}\left(b_{3}^{2} b_{6}\right), \\
\zeta^{2} b_{3}^{6}, v\left(\zeta^{2} b_{3}^{6}\right), & \zeta^{2} b_{3}^{4} b_{6}, v\left(\zeta^{2} b_{3}^{4} b_{6}\right) .
\end{array}
$$


12.2. Nonconnective fixed points. We can use the results of the previous section to determine the homotopy fixed-point spectrum for the action of $\mathbb{Z} / 2$ on $E$.

Due to the size and shape of the vanishing regions in the homotopy fixedpoint spectral sequences of the previous section, we observe that the localizations $\left(a_{1}^{-1} e\right)^{h \mathbb{Z} / 2}$ and $\left(a_{3}^{-1} e\right)^{h \mathbb{Z} / 2}$ have homotopy groups which are calculated by the localizations of the $E_{\infty}$ terms of the homotopy fixed-point spectral sequences. We write these as $b_{1}^{-1} \mathrm{TAF}^{D}$ and $b_{3}^{-1} \mathrm{TAF}^{D}$, though they are actually formed by inverting $b_{1}^{4}$ and $b_{3}^{8}$. The elements $\zeta^{8 k}$ and $\zeta^{8 k+6} b_{6}$ are destroyed in these localizations.

The homotopy groups of $b_{1}^{-1} \mathrm{TAF}^{D}$ form the ring which fits into the following pullback diagram.

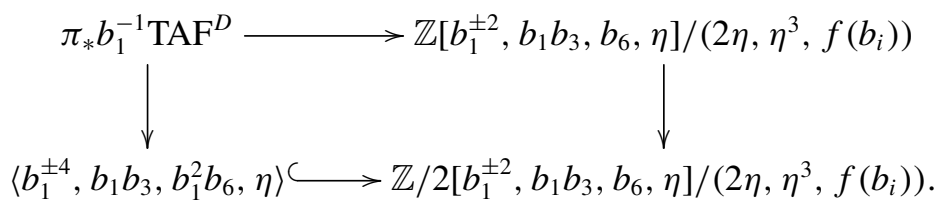

The homotopy groups of $b_{3}^{-1} \mathrm{TAF}^{D}$ are more convenient to study by introducing $y=b_{3}^{-2} b_{6}$, which is a degree-zero term satisfying a quadratic polynomial. The homotopy groups are free on $\{1, y\}$ over a subring $S$ which essentially coincides with the calculation of Mahowald and Rezk [MR09]. This subring lives in a short exact sequence $0 \rightarrow L \rightarrow S \rightarrow b_{3}^{-1} S \rightarrow 0$. Here $b_{3}^{-1} S$ fits into the pullback diagram

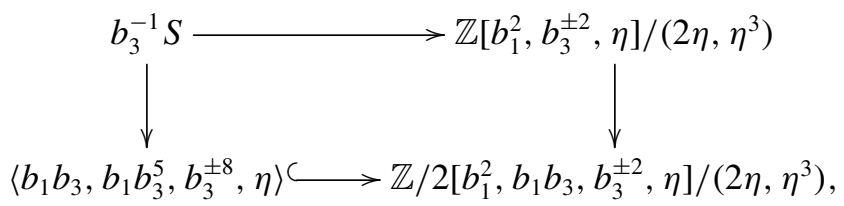

and $L$ is a free $\mathbb{Z} / 2\left[b_{3}^{ \pm 8}\right]$-module on the classes from Equation (12.1) that do not involve $b_{6}$.

The long exact sequence on homotopy groups induced by the homotopy pullback can then be employed to produce a description of the homotopy groups of $\mathrm{TAF}^{D}$. There is a short exact sequence $0 \rightarrow b_{3}^{-1} K \rightarrow \pi_{*} \mathrm{TAF}^{D} \rightarrow R^{\prime} \rightarrow 0$, where the ring $R^{\prime}$ agrees with $R$ in nonnegative degrees.

\section{Homotopy groups of $\left(\mathrm{TAF}^{D}\right)^{w_{15}}$}

In this section we compute the homotopy groups of $\mathrm{TAF}^{D}$ after taking homotopy fixed points with respect to the Atkin-Lehner operator $w_{15}$. Much 
like the previous section, this largely coincides with the Mahowald-Rezk computation, but adds some new $v_{1}$-periodic classes.

We first consider the homotopy fixed-point spectral sequence for the spectrum $e^{w_{15}}$; here $e$ is the connective spectrum of Proposition 11.1. Recall that $\omega \in \mathcal{O}_{K}$ is a primitive third root of unity.

PROPOSITION 13.1. The homotopy fixed-point spectral sequence for the homotopy groups of $e^{w_{15}}$ degenerates at $E_{2}=E_{\infty}$, with target

$$
\mathcal{O}_{K}\left[a_{1}, a_{3}, \tau\right] /\left(2 \tau,\left(a_{1}^{3}-\omega a_{3}\right)\left(a_{1}^{3}-\omega^{2} a_{3}\right) \tau\right)
$$

The homotopy fixed-point spectral sequences for $a_{1}^{-1} e, a_{3}^{-1} e$, and $\left(a_{1} a_{3}\right)^{-1} e$ degenerate in the same way.

Proof. By Proposition 10.2, the action of $w_{15}$ fixes $a_{1}$ and $a_{3}$, but sends $a_{6}$ to $-a_{6}-\left(a_{1}^{6}+a_{1}^{3} a_{3}+a_{3}^{2}\right)$. A direct calculation of the group cohomology gives the $E_{2^{-}}$ term described in Equation (13.1), where $\tau$ is the generator of $H^{2}(\mathbb{Z} / 2 ; \mathbb{Z})$. The terms are concentrated in even degrees, and so the spectral sequence collapses.

The corresponding calculation for the localizations follows in the same fashion.

Proposition 13.2. The class $\tau$ of Equation (13.1) lifts to a class in the homotopy of $e^{w_{15}}$ satisfying [2] $(\tau)=0$, where [2] $(x)$ is the 2-series of the formal group law of $e$.

Proof. The spectrum $e^{w_{15}}$, being a ring spectrum with homotopy concentrated in even degrees, is complex orientable; we may recast the resulting map $M U \rightarrow$ $e^{w_{15}}$ as a $\mathbb{Z} / 2$-equivariant map $M U \rightarrow e$, where $\mathbb{Z} / 2$ acts trivially on $M U$ and by the Atkin-Lehner involution $w_{15}$ on $e$. The resulting map on homotopy fixed points is a map $M U^{*}(B \mathbb{Z} / 2) \rightarrow \pi_{*} e^{w_{15}}$, and the class $\tau$ lifts to the orientation in $M U^{2}\left(\mathbb{C P}^{\infty}\right)$ whose image in $M U^{2}(B \mathbb{Z} / 2)$ satisfies [2] $(\tau)=0$.

COROLlary 13.3. The class $\tau$ lifts to an element in the homotopy of the spectrum $E^{w_{15}}$ on which $a_{1}$ and $a_{3}$ act invertibly.

Proof. We have that $\left(a_{1}^{6}+a_{1}^{3} a_{3}+a_{3}^{2}\right) \tau$ is zero in the homotopy fixed-point spectral sequences. After inverting $a_{3}$, the action of the element $a_{1}^{3} / a_{3}$ is as a third root of unity on $\tau$, and hence invertible. The element $a_{3} / a_{1}^{3}$ acts as a third root of unity after inverting $a_{1}$. As the action of $a_{1}$ and $a_{3}$ on $\tau$ are invertible after inverting any of $a_{1}, a_{3}$, or $a_{1} a_{3}$, it is so in $E^{w_{15}}$. 
COROLlaRY 13.4. We have an isomorphism

$$
\pi_{*} E^{w_{15}} \cong\left(E_{*}\right)^{w_{15}} \oplus\left(\mathcal{O}_{K}\left[a_{1}^{ \pm 1}\right]\right)^{2} \cdot \tau,
$$

We have $\tau^{2}=\alpha \tau$ for $\alpha$ some element congruent to $2 a_{1}^{-1} \bmod 4$.

Proof. By Corollary 13.3, the action of $a_{1}$ on $\tau$ is invertible, and by Proposition 13.2 we know that $\tau$ is annihilated by the 2 -series. We have

$$
[2](\tau)=2 \tau\left(1+\tau^{2} f_{0}\left(\tau^{2}\right)\right)+a_{1} \tau^{2}\left(1+\tau^{2} f_{1}\left(\tau^{2}\right)\right)
$$

for some power series $f_{0}$ and $f_{1}$, and this implies that

$$
\tau^{2}=a_{1}^{-1}(-2 \tau)\left(1+\tau^{2} g\left(\tau^{2}\right)\right)
$$

for some power series $g$. Applying the Weierstrass preparation theorem to $g$, we find that $\tau^{2}=\alpha \tau$ for some $\alpha$ congruent to $2 a_{1}^{-1} \bmod 4$.

Mod 2, the element $a_{3} / a_{1}^{3}$ acts as a third root of unity on $\tau$. By Hensel's lemma, there is a 2-adically convergent power series which is a unit multiple of $a_{3} / a_{1}^{3}$, acting as a third root of unity on $\tau$. This determines the action of the entire ring $\left(a_{1}^{-1} E_{*}\right)^{w_{15}}$ : in particular, it factors through $\mathcal{O}_{K}\left[a_{1}^{ \pm 1}\right](\omega) \cong\left(\mathcal{O}_{K}\left[a_{1}^{ \pm 1}\right]\right)^{2}$.

We now consider the homotopy fixed-point spectral sequence for the action of $\mathbb{Z} / 8$ on $E^{w_{15}}$. We have a comparison with the homotopy fixed-point spectral sequence for $E$, and we will now show that $\tau$ must support some nontrivial differential.

Proposition 13.5. The image of the class $\tau$ in the homotopy of $E^{w_{15}}$ does not lift to the homotopy of $\left(\mathrm{TAF}^{D}\right)^{w_{15}}$, or its localizations by $a_{1}, a_{3}$, or $\left(a_{1} a_{3}\right)$.

Proof. To show that $\tau$ does not lift to the homotopy fixed points of $\mathbb{Z} / 8 \times \mathbb{Z} / 2$ on $E$, we consider the $\mathbb{Z} / 2$-equivariant maps $\mathbb{S} \rightarrow \mathrm{TAF}^{D} \rightarrow E$, where the AtkinLehner involution acts on both $\mathrm{TAF}^{D}$ and $E$. The class $\tau$ appears as the unique generating class in filtration 2 and homotopy degree -2 in all three homotopy fixed-point spectral sequences, and in the homotopy fixed-point spectral sequence for $\mathrm{TAF}^{D}$ the only nonzero class in higher filtration is $\zeta^{14} b_{6}$.

The homotopy fixed-point spectral sequence for $\mathbb{S}$ calculates the stable cohomotopy groups of $\mathbb{R P}^{\infty}$, and in this spectral sequence the class $\tau$ supports a $d_{2}$-differential $d_{2}(\tau)=\eta \tau^{2}$ due to the attaching maps for the cells constructing $\mathbb{R P}^{\infty}$. As the map $\mathbb{S} \rightarrow \mathrm{TAF}^{D}$ is an inclusion of a summand in homotopy degrees -7 through 2 , the class $\tau$ also supports a $d_{2}$-differential in the homotopy fixed-point spectral sequence for $\left(\mathrm{TAF}^{D}\right)^{w_{15}}$.

The only class in higher filtration whose image could possibly be $\tau$ is $\zeta^{14} b_{6}$. However, this class is annihilated by inverting $a_{1}$, which acts invertibly on $\tau$. 
PROPOSITION 13.6. In the homotopy fixed-point spectral sequence for the action of $\mathbb{Z} / 8$ on $E^{w_{15}}$, we have a $d_{3}$-differential $d_{3}(\tau)=\eta^{3} b_{1}^{-2} \tau$. All remaining differentials are determined by their image in the homotopy fixed-point spectral sequence for $E$.

Proof. We first consider the $E_{3}$-page. The elements $\tau$ and $b_{1}^{2} \tau$ are in the kernel of the map to the $E_{3}$-page for $E$, and so can only support differentials to unit multiples of $\eta^{3} b_{1}^{-2} \tau$ or $\eta^{3} \tau$, respectively.

From the Leibniz rule either $\tau$ or $b_{1}^{2} \tau$ must support a nonzero differential. As the element $b_{3} / b_{1}^{3}$ is a cycle, we cannot have both of these differentials occurring. No matter which differential occurs, no elements in the kernel of the map of homotopy fixed-point spectral sequences survive above the 2-line, and so this is the only possible differential that $\tau$ could support. Therefore, we must have $d_{3}(\tau)=\eta^{3} b_{1}^{-2} \tau$ and $d_{3}\left(b_{1}^{2} \tau\right)=0$.

The $d_{3}$-differential is determined on classes which are not multiples of $\tau$ by their image in the homotopy fixed-point spectral sequence for $E$. On the $E_{4}$-page, the homotopy fixed-point spectral sequence for $E^{w_{15}}$ maps surjectively onto that for $E$, and the map is an isomorphism above the 2-line. Therefore, the remaining differentials are determined by their image in the homotopy fixed-point spectral sequence for $E$.

As a consequence of this description of the spectral sequence, we have the following.

THEOREM 13.7. Under the map

$$
\pi_{*}\left(\mathrm{TAF}^{D}\right)^{w_{15}} \rightarrow \pi_{*} \mathrm{TAF}^{D},
$$

the kernel is isomorphic to $\pi_{*}\left(\Sigma^{2} K O\right) \otimes W\left(\mathbb{F}_{4}\right)$, and the image in the homotopy of $\left(\mathrm{TAF}^{D}\right)^{w_{15}}$ consists of those classes in the homotopy of $\mathrm{TAF}^{D}$ which are not multiples of $b_{6}$.

\section{Acknowledgements}

The author would like to issue thanks to J. Voight for assistance with the fundamental domain, as well as general thanks toward M. Hill. The author was partially supported by NSF grant 1206008 and a fellowship from the Sloan foundation.

\section{References}

[AB04] M. Alsina and P. Bayer, 'Quaternion orders, quadratic forms, and Shimura curves', in: CRM Monograph Series, Vol. 22 (American Mathematical Society, Providence, RI, 2004). 
[BL10] M. Behrens and T. Lawson, 'Topological automorphic forms', Mem. Amer. Math. Soc. 204(958) (2010), xxiv+141.

[Eic38] M. Eichler, 'Über die Idealklassenzahl hyperkomplexer Systeme', Math. Z. 43(1) (1938), 481-494.

[Elk98] N. D. Elkies, 'Shimura curve computations', in: Algorithmic Number Theory, Portland, OR, 1998, Lecture Notes in Computer Science, 1423 (Springer, Berlin, 1998), 1-47.

[Goe09] P. G. Goerss, 'Realizing families of Landweber exact homology theories', in: New Topological Contexts for Galois Theory and Algebraic Geometry (BIRS 2008), Geom. Topol. Monogr., 16 (Geom. Topol. Publ., Coventry, 2009), 49-78.

[HL10] M. Hill and T. Lawson, 'Automorphic forms and cohomology theories on Shimura curves of small discriminant', Adv. Math. 225(2) (2010), 1013-1045.

[KRY06] S. S. Kudla, M. Rapoport and T. Yang, 'Modular forms and special cycles on Shimura curves', in: Annals of Mathematics Studies, Vol. 161 (Princeton University Press, Princeton, NJ, 2006).

[Kur79] A. Kurihara, 'On some examples of equations defining Shimura curves and the Mumford uniformization', J. Fac. Sci. Univ. Tokyo Sect. IA Math. 25(3) (1979), 277-300.

[Lan76] P. S. Landweber, 'Homological properties of comodules over $M \mathrm{U}_{*}(M \mathrm{U})$ and $B P_{*}($ B P)', Amer. J. Math. 98(3) (1976), 591-610.

[Mic81] J.-F. Michon, 'Courbes de Shimura hyperelliptiques', Bull. Soc. Math. France 109(2) (1981), 217-225.

[Miy89] T. Miyake, Modular Forms, (Springer, Berlin, 1989), (translated from the Japanese by Y. Maeda).

[Mor81] Y. Morita, 'Reduction modulo P of Shimura curves', Hokkaido Math. J. 10(2) (1981), 209-238.

[MR09] M. Mahowald and C. Rezk, 'Topological modular forms of level 3', Pure Appl. Math. Q. 5(2) (2009), 853-872. (special issue: in honor of Friedrich Hirzebruch. Part 1).

[Qui69] D. Quillen, 'On the formal group laws of unoriented and complex cobordism theory', Bull. Amer. Math. Soc. 75 (1969), 1293-1298.

[Vig80] M.-F. Vignéras, Arithmétique des algèbres de quaternions, Lecture Notes in Mathematics, 800 (Springer, Berlin, 1980). 